\title{
Improved algorithm for computing the domain of attraction of rational nonlinear systems
}

\author{
Péter Polcz ${ }^{\mathrm{a}}$, Tamás Péni ${ }^{\mathrm{b}}$, Gábor Szederkényi ${ }^{\mathrm{a}, \mathrm{b}}$ \\ ${ }^{a}$ Faculty of Information Technology and Bionics, Pázmány Péter Catholic University, \\ Práter u. 50/a, H-1083 Budapest, Hungary \\ ${ }^{b}$ Systems and Control Laboratory, Institute for Computer Science and Control (MTA \\ SZTAKI), Hungarian Academy of Sciences, Kende u. 13-17, H-1111 Budapest, Hungary
}

\begin{abstract}
An improved method for computing a bounded estimate of the domain of attraction (DOA) of locally asymptotically stable uncertain rational nonlinear system models is proposed in this paper. The approach is based on the previous work of Trofino and Dezuo (2013). Using linear fractional transformation and an additional simplification step, we give a novel automatic method for generating the rational terms to be considered in the Lyapunov function, which satisfy the requirements for system representation. Moreover, we give an algorithm for computing the so-called maximal annihilators, which contain the maximum number of linearly independent rows corresponding to a given feasibility domain. As the illustrative examples show, the proposed method effectively reduces the size of the resulting optimization problem without increasing the conservatism of the DOA computation.
\end{abstract}

Keywords: nonlinear systems, uncertain systems, stability, Lyapunov functions, domain of attraction, linear matrix inequalities, 2000 MSC: 37B25

\section{Introduction}

Approximating the domain of attraction (DOA) is often a fundamental task in model analysis and controller design/evaluation. The stability proper-

\footnotetext{
Email addresses: polcz.peter@itk.ppke.hu (Péter Polcz), peni.tamas@sztaki.mta.hu (Tamás Péni), szederkenyi@itk.ppke.hu (Gábor Szederkényi)
} 
ties of dynamical systems are most often studied using Lyapunov functions, accordingly, the computational construction of Lyapunov functions [2] has been addressed extensively in the literature.

It is well-known that the DOA of an asymptotically stable equilibrium point of a dynamical system $\dot{x}=f(x), x \in \mathbb{R}^{n}$ can be precisely determined in theory by solving Zubov's first order nonlinear partial differential equation [3]. There exist several generalizations of Zubov's method, such as [4] that is dedicated to determine the robust domain of attraction of an uncertain system $\dot{x}(t)=f(x(t), \delta(t))$, where $\delta: \mathbb{R} \mapsto \mathcal{D}$ is a bounded perturbation and $\mathcal{D}$ is a compact subset of $\mathbb{R}^{d}$, and it is assumed that $f(0, \delta)=0$ for all $\delta$ in $\mathcal{D}$. The main disadvantage of this method is that the solvability of Zubov's partial differential equation cannot be foreseen.

Another fundamental result in this field is the existence of so-called maximal Lyapunov functions for a wide class of nonlinear systems and the corresponding partial differential equation which characterizes them [5]. In comparison with Zubov's equation, an iterative procedure is given for approximating the maximal rational Lyapunov function. An algorithm for generating Lyapunov functions for a special class of nonlinear systems based on the construction of polytopes is given in [6]. In [7], a linear programming based method is given for the construction of Lyapunov functions for general planar nonlinear systems. In [8], maximal Lyapunov functions were defined and computed for hybrid (piecewise nonlinear) systems.

Although the above mentioned nonlinear (rational) structures are advantageous for DOA computation, they are less attractive from a numerical point of view. Generally, the corresponding computations result in complex (often nonlinear) optimization problems, which are difficult to solve. In order to make these procedures numerically tractable, several new approaches have been developed in the last years. For example, the use of linear matrix inequalities (LMIs) and semidefinite programming (SDP) techniques for nonlinear systems has become very popular due to their advantageous properties and the availability of efficient numerical tools to solve such problems (see, e.g. $[9,10])$. These efficient techniques provide a powerful framework for stability analysis, robust control and filtering problems. Ghaoui et.al. [11] used quadratic Lyapunov functions and linear fractional transformations (LFT) to represent rational nonlinear systems and defined convex conditions for stability analysis and state feedback design. Methods applying sum of squares (SOS) programming to maximize the estimate of the region of attraction can be found in $[2,12]$. Stability conditions in previously mentioned works are 
converted into LMIs using SOS relaxations and the generalization of the Sprocedure. In [26], rational Lyapunov functions have been used for uncertain polynomial systems. Starting from a given initial function, an optimal one is computed for which the necessary properties are ensured by an appropriate non-convex bilinear matrix inequality (BMI). Under certain conditions, the BMI can be reformulated as a quasi-convex generalized eigenvalue problem.

A recent important result from this line of research is published in [1], where the authors use Finsler's lemma and the notion of annihilators to compute rational Lyapunov functions for a wide class of locally asymptotically stable nonlinear systems. The newly introduced sufficient conditions for stability are affine parameter dependent LMIs obtained from the prescribed properties of Lyapunov functions. The choice of the affine annihilator plays an important role in the LMI conditions, since it represents the algebraic relations between the elements of the chosen set of monomial and rational functions to appear in the Lyapunov function. The paper [1] primarily focuses on the fundamental theory of DOA computation using Finsler's lemma. Therefore, there is a room for the further study of the automatic generation of the set of rational functions contained in the Lyapunov function and that of a corresponding annihilator. In case of polynomial systems, the set of monomials, which are collected in the vector $\pi$, is set to contain all monomials of degree less than or equal to the maximal degree term in the system equation. This generally causes a combinatorial explosion as the number of variables and their degrees increase. As a resolution, it is proposed to skip certain monomials from the model, e.g. if a state variable does not appear in a nonlinear term of the system equation, monomials of the respective variable are advised to be omitted from $\pi$.

In general, three sources of freedom exists in the problem formulation, which directly affect the size and shape of the estimated DOA:

(a) Due to the fact that the differential-algebraic representation of the system is not unique, we are able to select the set of rational functions in several ways. This set of rational functions (denoted by $\pi$ ) represents the rational uncertain terms in the system equation. The structure of the Lyapunov function is chosen to be a quadratic form of the state variables and these nonlinear uncertain rational terms. In fact, any additional new function in $\pi$ brings further degrees of freedom in the Lyapunov function computation problem, which may result in a better estimate. However, the dimensions of the corresponding optimization problem will increase 
combinatorially with the added terms.

(b) The second source of freedom is the structure of the affine annihilator of $\pi$. It is clear from the problem definition that the annihilator corresponding to a given Lyapunov function computation is non-unique, but there are no results in the literature, how to construct a "good" affine annihilator.

(c) Finally, a bounded polytopic domain should be defined, into which the state variables are constrained to belong. The feasibility of the LMIs corresponding to the Lyapunov conditions are checked only in the interior of this polytope.

It is important to add that the estimation of the DOA has wide theoretical and practical application possibilities. For example, in [13], the author shows that there is an interesting trade-off between the domain of attraction and the convergence rate of extremum seeking control. In [14], a stabilizing controller is designed for an experimental cart-pendulum system using the method of Immersion and Invariance, and it is shown through DOA analysis that the closed loop system is stable for any initial position of the pendulum in the upper-half plane.

Based on the above, the purpose of this paper is to propose novel automatic model and annihilator generation steps for the method in [1], that efficiently reduce the size of the resulting optimization problem without increasing significantly the conservatism of the DOA computation.

The paper is organized as follows: Section 2 summarizes the LMI-based algorithm for computing the DOA of rational nonlinear systems proposed in [1]. In the next section, we propose an LFT based method to construct a reduced set of rational functions to appear in the Lyapunov function. In Section 4, we present a new method for annihilator selection. In the last section, three illustrative examples are shown.

\subsection{Notations, abbreviations}

In this paper, we will use the following notations and abbreviations: $i=$ $\overline{1, n}$ denotes that $i \in\{1, \ldots, n\} .0_{n \times m}$ and $I_{n}$ denote the $n \times m$ zero matrix and the $n \times n$ unit matrices, respectively. $\mathcal{S}^{m}:=\left\{X \in \mathbb{R}^{m \times m} \mid X=X^{T}\right\}$ denotes the cone of symmetric matrices. $A \succ 0$ and $A \prec 0$ denotes that $A \in \mathcal{S}^{m}$ is positive and negativity definite, respectively. Given a scalar valued positive definite proper function $V: \mathbb{R}^{n} \rightarrow \mathbb{R}$, its particular level set

$\varepsilon_{\alpha}=\left\{x \in \mathbb{R}^{n}: V(x) \leq \alpha\right\}$ is said to be the $\alpha$-level set of function $V$. A 
positive definite function is called proper if $\varepsilon_{\alpha}$ is a compact set for all $\alpha>0$. Function $f \in \mathbb{R}^{n} \rightarrow \mathbb{R}$ is called rational if $f(x):=f\left(x_{1}, \ldots, x_{n}\right)=\frac{p(x)}{q(x)}$, where $p(x)$ and $q(x)$ are polynomials of the variables $x_{1}, \ldots, x_{n}$. A rational function $f$ is said to be well-defined on $\mathcal{X} \subseteq \mathbb{R}^{n}$ if $q(x) \neq 0$ for all $x \in \mathcal{X}$. We call a vector valued function $f \in \mathbb{R}^{n} \rightarrow \mathbb{R}^{m}$ rational (and well-defined on $\mathcal{X}$ ) if its coordinates functions $f_{i}(x)$ are rational functions (that are well defined on $\mathcal{X}), i=\overline{1, m}$.

\section{LMI-based algorithm for DOA computation}

Following the concept of [1], we consider nonlinear systems of the form

$$
\dot{x}(t)=f(x(t), \delta) \quad x(t) \in \mathbb{R}^{n}, x_{0} \in \mathcal{X}, \delta \in \mathcal{D}
$$

where $x(t) \in \mathbb{R}^{n}$ is the state vector, $x(0)=x_{0}$ is the initial condition, and $\delta \in \mathbb{R}^{d}$ is a vector of constant uncertain parameters. $\mathcal{X} \subset \mathbb{R}^{n}$ and $\mathcal{D} \subset \mathbb{R}^{d}$ are bounded polytopes given a priori. We assume that the origin $0 \in \mathbb{R}^{n}$ is inside $\mathcal{X}$, which is the set of initial states considered in the stability analysis. Moreover, it is assumed that $f: \mathcal{X} \times \mathcal{D} \rightarrow \mathbb{R}^{n}$ is a vector valued function of $(x, \delta) \in \mathcal{X} \times \mathcal{D} \subset \mathbb{R}^{n+d}$ having the form

$$
f(x, \delta)=\left(\begin{array}{c}
f_{1}(x, \delta) \\
\ldots \\
f_{n}(x, \delta)
\end{array}\right), \quad f_{i}(x, \delta)=\sum_{j=1}^{M_{i}} \frac{p_{i j}(x, \delta)}{q_{i j}(x, \delta)}, \quad i=\overline{1, n}
$$

where $f_{i}: \mathbb{R}^{n+d} \rightarrow \mathbb{R}$ are well-defined rational scalar functions, i.e. $p_{i j}(x, \delta)$ and $q_{i j}(x, \delta)$ are polynomials of $(x, \delta)$ and $q_{i j}(x, \delta) \neq 0$ for all $(x, \delta) \in \mathcal{X} \times \mathcal{D}$. Finally, we assume that the origin $x^{*}=0$ is an asymptotically stable equilibrium point of (1) for all $\delta \in \mathcal{D}$.

The set of all initial conditions, from which the solutions converge to $x^{*}$ is called the domain of attraction (DOA). Clearly, the DOA of system (1) can be estimated by an appropriate level set of the local Lyapunov function $V(x, \delta)$.

Remark 2.1. Even though the true DOA of $x^{*}$ might be unbounded, the method proposed in this paper assumes that $\mathcal{X}$ and $\mathcal{D}$ are bounded polytopes. Therefore, the computed stability region, which should be located entirely in the interior of $\mathcal{X}$, is always bounded. 
In order to construct a suitable Lyapunov function, [1] proposes to start from the following differential algebraic representation of (1):

$$
\begin{aligned}
& \dot{x}(t)=f(x(t), \delta)=A x(t)+B \pi(x(t), \delta) \quad x(t) \in \mathbb{R}^{n}, \quad \delta \in \mathbb{R}^{d} \\
& 0=N_{b}(x, \delta) \cdot\left(\begin{array}{c}
x \\
\pi(x, \delta)
\end{array}\right) \quad \forall(x, \delta) \in \mathbb{R}^{n+d} \quad \pi(x, \delta) \in \mathbb{R}^{p}
\end{aligned}
$$

where $A \in \mathbb{R}^{n \times n}, B \in \mathbb{R}^{n \times p}$ are constant matrices, and $\pi: \mathbb{R}^{n+d} \rightarrow \mathbb{R}^{p}$ is a vector valued function in which the coordinates are smooth distinct nonlinear rational functions of $(x, \delta)$. For convenience, we denote the combined vector $\left(\begin{array}{c}x \\ \pi(x, \delta)\end{array}\right)$ by $\pi_{b}(x, \delta) \in \mathbb{R}^{m}$. Finally, $N_{b}: \mathbb{R}^{n+d} \rightarrow \mathbb{R}^{q \times m}$ is an affine matrix function of $(x, \delta)$, and it is called an affine annihilator of $\pi_{b}(x, \delta)$ due to the equality (3b). From now on, the arguments of $x, \pi$ and $\pi_{b}$ will be suppressed. To summarize, variables $n, d, p$ and $m=n+p$ denote the dimensions of $x$, $\delta, \pi$ and $\pi_{b}$, respectively, and $q$ is the number of rows of annihilator $N_{b}(x, \delta)$. Remark 2.2. If $f(0, \delta)=0$ for all $\delta \in \mathcal{D}$, then we can allow $\delta(t)$ to vary smoothly in time, and we assume that $\dot{\delta}(t) \in \check{\mathcal{D}}$, where $\check{\mathcal{D}}$ is a bounded polytopic set. For smooth time-variant uncertain parameters (with bounded derivatives) the same optimization model [1] can be built. The reason we restrict the uncertain parameter to be constant is that we consider systems $\dot{\bar{x}}=g(\bar{x}, \delta)$ having a nontrivial equilibrium point $\bar{x}^{*}(\delta)$, which depends (smoothly) on the actual value of $\delta$. In order to center the system, we introduce the centered state vector $x=\bar{x}-\bar{x}^{*}(\delta)$. Then, considering the time derivative of the new state vector, we obtain the equation of the centered system as

$$
\dot{x}=f(x, \delta):=g\left(x+\bar{x}^{*}(\delta), \delta\right)-\frac{\partial \bar{x}^{*}(\delta)}{\partial \delta} \dot{\delta}
$$

Assuming that the uncertain parameters are constant in time, the second term $\partial \bar{x}^{*}(\delta) / \partial \delta \dot{\delta}$ vanishes. Since we have that $g\left(\bar{x}^{*}(\delta), \delta\right)=0$ for all $\delta \in \mathcal{D}$, the origin is an equilibrium point of the system in Eq. (4).

The candidate rational Lyapunov function is assumed to be in the following quasi-quadratic form

$$
V(x, \delta)=\pi_{b}^{T} P_{b} \pi_{b}, \quad \pi_{b}=\left(\begin{array}{l}
x \\
\pi
\end{array}\right)
$$

where $P_{b} \in \mathcal{S}^{m}$ is a constant symmetric matrix, (not necessarily positive definite). For $V$ to be a local Lyapunov function, it must fulfill the following 
conditions:

$$
\begin{array}{ll}
v_{l}(\|x\|) \leq V(x, \delta) \leq v_{u}(\|x\|) & \forall(x, \delta) \in \mathcal{X} \times \mathcal{D} \\
\dot{V}(x, \delta):=\frac{\partial V(x, \delta)}{\partial x} f(x, \delta) \leq-v_{d}(\|x\|) & \forall(x, \delta) \in \mathcal{X} \times \mathcal{D}
\end{array}
$$

where $v_{l}(\cdot), v_{u}(\cdot)$ and $v_{d}(\cdot)$ are continuous strictly increasing functions, which are zero in $\|x\|=0$. Since there is an interdependence between the coordinates of $\pi_{b}$, constraining $P_{b}$ to be positive definite is a more conservative restriction than stating that the Lyapunov function $V(x, \delta)=\pi_{b}^{T} P_{b} \pi_{b}$ is positive for every $(x, \delta) \in \mathcal{X} \times \mathcal{D}$. Consequently, several possible Lyapunov function candidates would be excluded from the optimization. Using the notion of annihilators and a special case of Finsler's lemma [1, 15, 16], less conservative sufficient matrix inequality conditions are formulated in [1], which imply the Lyapunov conditions.

Let $\Omega \subset \mathbb{R}^{s}$ be a bounded polytope, and $z(\omega) \in \mathbb{R}^{m}$ a well-defined rational function of $\omega \in \Omega$ with $N: \Omega \rightarrow \mathbb{R}^{q \times m}$ being its affine annihilator, i.e. $N(\omega) z(\omega)=0$ for all $\omega \in \mathbb{R}^{s}$. Assume that there exists a symmetric matrix $P \in \mathcal{S}^{m}$ and matrix $L \in \mathbb{R}^{m \times q}$ such that

$$
\mathcal{L}_{N}(P, L):=P+L N(\omega)+N^{T}(\omega) L^{T} \succ 0, \forall \omega \in \Omega,
$$

Then, as a consequence of Finsler's lemma, function $z(\omega)^{T} P z(\omega)$ is strictly positive for all $\omega \in \Omega \backslash\{0\}$. Inequality (7) is a parameter $(\omega)$ dependent LMI, for which the parameter vector $\omega$ belongs to a bounded polytope $\Omega$, therefore, (7) can be numerically handled by checking its feasibility in $\mathcal{V}(\Omega)$, where $\mathcal{V}(\Omega)$ denotes the corner points of $\Omega$ [17, Proposition 5.4] .

Thus, for the positivity of the Lyapunov function $V(x, \delta)=\pi_{b}^{T} P_{b} \pi_{b}$, one can formulate a sufficient parameter dependent LMI condition

$$
\mathcal{L}_{N_{b}}\left(P_{b}, L_{b}\right) \succ 0, \forall(x, \delta) \in \mathcal{V}(\mathcal{X} \times \mathcal{D}),
$$

with an appropriate annihilator $N_{b}(x, \delta)$ for $\pi_{b}$. Since the time derivative of the Lyapunov function can be altered to the form

$$
\dot{V}(x, \delta)=\pi_{a}^{T}\left(P_{a}+P_{a}^{T}\right) \pi_{a},
$$

its negativity can be ensured by the condition

$$
\mathcal{L}_{N_{a}}\left(-P_{a}-P_{a}^{T}, L_{a}\right) \succ 0, \forall(x, \delta) \in \mathcal{V}(\mathcal{X} \times \mathcal{D}),
$$


where the matrix $P_{a}$ and function $\pi_{a}$ are defined in [1, Eqs. $\left.(39,57)\right]$, and $N_{a}(x, \delta)$ is an affine annihilator of $\pi_{a}$, for which a possible selection can be derived from annihilator $N_{b}(x, \delta)$ as presented in [1, Eqs. $\left.(40,43)\right]$.

In order that the 1-level set approach the boundaries of $\mathcal{X}$ as much as possible (see, [1]), the value of the Lyapunov function on each facet $\mathcal{F}_{k}$ of the polytope $\mathcal{X}$ is prescribed to be between the values: $1 \leq V(x, \delta) \leq \tau_{k}$, for all $(x, \delta) \in \mathcal{F}_{k} \times \mathcal{D}, k=\overline{1, M_{\mathcal{X}}}$, where $\tau_{k}$ are free variables and are meant to be minimized through the optimization procedure. Furthermore, $M_{\mathcal{X}}$ denotes the number of facets of $\mathcal{X}$. To briefly explain this approach, consider the case when $1 \leq V(x, \delta) \leq 1+\epsilon_{k}$, where $\epsilon_{k}$ are small positive values for $k=\overline{1, M_{\mathcal{X}}}$. Then, the 1-level set almost matches the boundary of $\mathcal{X}$. Using another special case of Finsler's lemma, these conditions can be expressed as affine parameter dependent LMI conditions [1, Section 5, Eqs. (89) and (90)].

In the next section, we present a method to construct a reduced set of rational functions (i.e. a possible $\pi$ vector with preferably few entries) to be considered in the Lyapunov function. In Section 4, a maximal annihilator $(N(\omega))$ selection algorithm is presented for a certain rational function $z(\omega) \in \mathbb{R}^{m}$.

\section{Constructing a set of rational functions using LFR}

In this section, we present our approach for an automatic method to generate a set of nonlinear rational functions $(\pi)$, by using LFT and further systematic algebraic model simplification steps. In general, the obtained set of functions leads to a dimensionally reduced optimization problem compared to other known solutions in the literature, since fewer rational terms are considered in the structure of the Lyapunov function.

$\mathrm{LFT}^{1}$ plays an important role in modeling uncertain rational systems, and it is often used in the literature [12], as presented in [11]. Using LFT, the linear and nonlinear part of any autonomous quasi linear parameter varying (quasi-LPV or qLPV) system of the form $\dot{x}=\mathcal{A}(x, \delta) x$ can be separated as

\footnotetext{
${ }^{1}$ The LFT is discussed in detail e.g. in the book [18, Chapter 10.].
} 


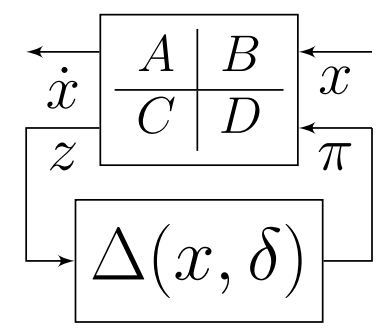

Figure 1: Block-diagram of the linear fractional representation (LFR)

follows:

$$
\begin{aligned}
& \left(\begin{array}{l}
\dot{x} \\
z
\end{array}\right)=\left(\begin{array}{ll}
A & B \\
C & D
\end{array}\right) \cdot\left(\begin{array}{l}
x \\
\pi
\end{array}\right) \leftrightarrow \begin{array}{l}
\dot{x}=A x+B \pi, \\
z=C x+D \pi,
\end{array} \quad \begin{array}{l}
x \in \mathbb{R}^{n} \\
z \in \mathbb{R}^{p}
\end{array} \\
& \pi=\Delta(x, \delta) z \quad z \in \mathbb{R}^{p}
\end{aligned}
$$

where $A \in \mathbb{R}^{n \times n}, B \in \mathbb{R}^{n \times p}, C \in \mathbb{R}^{p \times n}, D \in \mathbb{R}^{p \times p}$ are constant matrices and $\Delta: \mathbb{R}^{n+d} \rightarrow \mathbb{R}^{p \times p}$ is a (not necessarily diagonal) matrix function of the state variables and of the uncertain parameters. Figure 1 illustrates the block diagram of the system representation in Eq. (11). Equation (11a) can be considered as a linear time invariant (LTI) system with an input defined by the nonlinear uncertain function shown in Eq. (11b). Multiplying the second equation of (11a) by $\Delta(x, \delta)$ from the left, and using equation (11b), we obtain that $G(x, \delta) x+F(x, \delta) \pi=0$ where $G(x, \delta):=-\Delta(x, \delta) C \in \mathbb{R}^{p \times n}$ and $F(x, \delta):=I_{p}-\Delta(x, \delta) D \in \mathbb{R}^{p \times p}$. We assume that this representation is well-defined, namely $F(x, \delta)$ is invertible for all $(x, \delta) \in \mathcal{X} \times \mathcal{D}$, (i.e. the linear fractional representation (LFR) is well-posed [18, Definition 10.2]). Then we can give a formula for $\pi(x, \delta)=-[F(x, \delta)]^{-1} G(x, \delta) x$.

\subsection{Model simplification steps}

In general, the terms in $\pi$ can be generated by the available symbolic computation tools. When using e.g. the LFR toolbox [19, 20], $\pi$ contains rational functions, in which a certain basis term $\frac{p(x, \delta)}{r(x, \delta)}$ may appear several times in $\pi$, thus increasing the dimension of the model.

Example 3.1. Consider a simple one dimensional system $\dot{x}=\frac{x(x+1)}{r(x)}$, with $r(x)=x^{4}+x^{2}+1$. Function sym2lfr of the LFR-toolbox gives a model in which the following nonlinear terms appear in $\pi$ :

$$
\pi^{T}=\left(\begin{array}{ccccc}
\frac{x^{2}}{r(x)} & \frac{x^{3}+x^{5}}{r(x)} & \frac{x^{2}+x^{4}}{r(x)} & \frac{x^{3}}{r(x)} & \frac{x^{2}}{r(x)}
\end{array}\right)
$$


We can observe that the terms $\frac{x^{3}}{r(x)}$ and $\frac{x^{2}}{r(x)}$ appear several times in $\pi$.

Using the exact 1-d order reduction technique ${ }^{2}$ [21], one can obtain a reduced LFR, however, certain rational terms may disappear from $\pi$, which can lead to more conservative estimates. From a computational point of view, the produced rational functions in $\pi$ may contain fairly complex expressions, for which the symbolic computations take significant effort and processing time.

To overcome the above mentioned problems, we present an algebraic procedure for model simplification, which guarantees that no rational terms are eliminated from the initial $\pi$. We propose altering the form of $\pi$ into a normal form, in which both the monomial numerator and the polynomial denominator are monic (with leading coefficient 1), and no repetitive terms appear in $\pi$. We should note that any modifications of $\pi$ requires $B$ to be adapted as well, in order to preserve the value of the term $B \pi$ in Eq. (3a). We propose the following three auxiliary algebraic steps, to transform $\pi$ into its normal form $^{3}$. For convenience, we illustrate the transformation steps with simple examples.

1. Split up the polynomial numerators appearing in $\pi$ into monic monomials.

For a $2 \times 1$ matrix $B$ and a corresponding rational term $\frac{\alpha p(x, \delta)+\beta q(x, \delta)}{r(x, \delta)}$, this step works as follows:

$$
B \pi=\left(\begin{array}{l}
b_{1} \\
b_{2}
\end{array}\right)\left(\frac{\alpha p(x, \delta)+\beta q(x, \delta)}{r(x, \delta)}\right) \Longrightarrow\left(\begin{array}{ll}
\alpha b_{1} & \beta b_{1} \\
\alpha b_{2} & \beta b_{2}
\end{array}\right)\left(\begin{array}{l}
\frac{p(x, \delta)}{r(x, \delta)} \\
\frac{q(x, \delta)}{r(x, \delta)}
\end{array}\right)=B^{(1)} \pi^{(1)}
$$

where $\alpha, \beta$ are real numbers, $p(x, \delta)$ and $q(x, \delta)$ are monic monomials, and $r(x, \delta)$ is a polynomial of $x$ and $\delta$.

2. Scale each coordinate of $\pi^{(1)}$, such that both the monomial numerators and polynomial denominators be monic.

On the simple example of the previous step, this transformation can

\footnotetext{
${ }^{2}$ The computation was performed using the LFR-toolbox function minlfr1.

${ }^{3}$ The presented algebraic manipulation is automated using Matlab's Symbolic Math Toolbox (Symbolic-toolbox).
} 
be illustrated as:

$$
B^{(1)} \pi^{(1)}=\left(\begin{array}{ll}
b_{11} & b_{12} \\
b_{21} & b_{22}
\end{array}\right)\left(\begin{array}{l}
\alpha^{\prime} \cdot \frac{p(x, \delta)}{r^{\prime}(x, \delta)} \\
\beta^{\prime} \cdot \frac{q(x, \delta)}{r^{\prime}(x, \delta)}
\end{array}\right) \Longrightarrow\left(\begin{array}{ll}
\alpha^{\prime} b_{11} & \beta^{\prime} b_{12} \\
\alpha^{\prime} b_{21} & \beta^{\prime} b_{22}
\end{array}\right)\left(\begin{array}{l}
\frac{p(x, \delta)}{r^{\prime}(x, \delta)} \\
\frac{q(x, \delta)}{r^{\prime}(x, \delta)}
\end{array}\right)=B^{(2)} \pi^{(2)},
$$

where $\alpha^{\prime}, \beta^{\prime}$ are real numbers, and both monomials $p(x, \delta)$ and $q(x, \delta)$, and polynomial $r^{\prime}(x, \delta)$ are monic.

3. Eliminate repetitive terms in $\pi^{(2)}$ and merge (add) the corresponding columns of $B$.

Assume that a rational term $w(x, \delta)=\frac{p(x, \delta)}{r^{\prime}(x, \delta)}$ with a monic monomial numerator and monic polynomial denominator appears twice in the equations with a $2 \times 2$ coefficient matrix $B^{(2)}$. Then this step is performed as:

$$
B^{(2)} \pi^{(2)}=\left(\begin{array}{ll}
b_{11}^{\prime} & b_{12}^{\prime} \\
b_{21}^{\prime} & b_{22}^{\prime}
\end{array}\right)\left(\begin{array}{l}
w(x, \delta) \\
w(x, \delta)
\end{array}\right) \Longrightarrow\left(\begin{array}{l}
b_{11}^{\prime}+b_{12}^{\prime} \\
b_{21}^{\prime}+b_{22}^{\prime}
\end{array}\right)(w(x, \delta))=B^{(3)} \pi^{(3)} .
$$

The above three steps clearly guarantee that $\pi^{(3)}$ will be in the required form. From now on, we assume that $\pi$ is in normal form.

\section{A novel method for annihilator selection}

As already presented in Section 2, the positivity of a rational function in the general quadratic form $z(\omega)^{T} P z(\omega)$ is ensured by a sufficient parameter dependent LMI condition (7), which can allow even indefinite matrices for $P$. In LMI (7), the annihilator $N(\omega)$ represents the algebraic relation between the coordinates of $z(\omega)$. Therefore, the set of possible values of $P$ for which $z(\omega)^{T} P z(\omega)$ is positive is highly dependent on the choice of annihilator $N(\omega)$. In this section, we propose a systematic method for annihilator generation for a fixed well-defined vector valued rational function $z(\omega)$, which is later applied on the fixed functions $\pi_{b}(x, \delta)$ and $\pi_{a}(x, \delta)$ corresponding to the rational basis of the Lyapunov function and its time derivative, respectively.

Let $\mathfrak{F}_{1} \subset \mathcal{S}^{m}$ denote the set consisting of every possible symmetric matrix $P$, for which the condition

$$
z(\omega)^{T} P z(\omega)>0, \forall \omega \in \Omega \backslash\{0\}
$$


is satisfied. In general, (16) is difficult to be numerically tested, but $\mathfrak{F}_{1}$ can be approximated by LMI conditions. Let $\mathfrak{F}_{0}$ be the set of positive definite $m \times m$ symmetric matrices. Then it is clear that $\mathfrak{F}_{0}$ is a subset of $\mathfrak{F}_{1}$.

Now we consider the more permissive (i.e., less conservative) parameter dependent LMI condition (7) with an annihilator $N: \mathbb{R}^{s} \rightarrow \mathbb{R}^{q \times m}$, such that $N(\omega) z(\omega)=0$, for all $\omega \in \mathbb{R}^{s}$. The feasible set (i.e., solution set) of matrix inequality (7) corresponding to annihilator $N$ is denoted by

$$
\mathfrak{F}_{N}=\left\{P \in \mathcal{S}^{m} \mid \exists L \in \mathbb{R}^{m \times q} \text { s.t. } \mathcal{L}_{N}(P, L) \succ 0, \forall \omega \in \Omega\right\}
$$

We emphasize that $L$ is also a free matrix variable of the LMI condition in Eq. (7) and hence that of the optimization problem. However, $L$ is not present in the construction of function $z(\omega)^{T} P z(\omega)$, and its actual value is unimportant. Therefore, the feasible set $\mathfrak{F}_{N} \subset \mathcal{S}^{m}$ is related only to the possible values of $P$. As a consequence of Finsler's lemma, we can say that for every $P \in \mathfrak{F}_{N}$, function $z(\omega)^{T} P z(\omega)$ is positive definite. Formally, $\mathfrak{F}_{0} \subseteq \mathfrak{F}_{N} \subseteq \mathfrak{F}_{1}$.

\subsection{Row reduced equivalent of an annihilator}

In this subsection, we will show that in certain cases an annihilator can be redundant, in the sense that another annihilator with less rows can generate the same feasible set for inequality (7).

Let us introduce an auxiliary object, the coefficient matrix of an affine annihilator, which will ease further notations and operations. Since $N(\omega)$ is an affine matrix function of $\omega$, each element of it can be expressed as a first order polynomial of the form:

$$
N_{l i}(\omega)=\left(\vartheta_{l, i 0}+\sum_{j=1}^{s} \vartheta_{l, i j} \omega_{j}\right), \quad \begin{aligned}
& l=\overline{1, q} \\
& i=\overline{1, m}
\end{aligned}
$$

where $\vartheta_{l, i j} \in \mathbb{R}$ are the coefficients of the affine terms $1, \omega_{j}$ in the $l$ th row and $i$ th column of $N(\omega)$. The $l$ th row of $N(\omega)$ can be represented by the row vector $\vartheta_{l} \in \mathbb{R}^{1 \times m(s+1)}$. Considering these coefficients, we can construct a so-called coefficient matrix $\Theta_{N} \in \mathbb{R}^{q \times m(s+1)}$ for $N(\omega)$ :

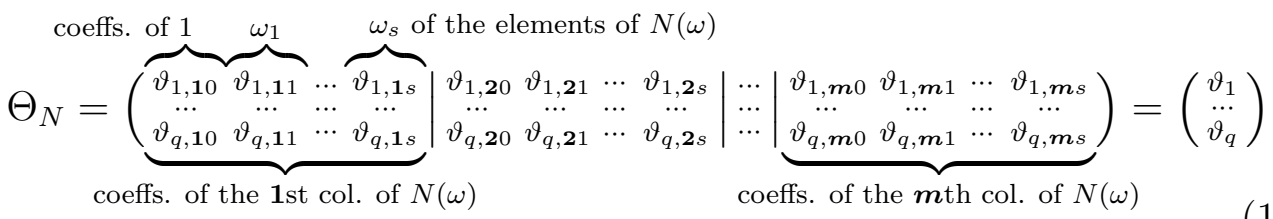


This coefficient matrix can uniquely determine the corresponding annihilator by using the following formula: $N(\omega)=\Theta_{N} \cdot\left(I_{m} \otimes\left(\begin{array}{c}1 \\ \omega\end{array}\right)\right)$, where $\otimes$ denotes the Kronecker product. Therefore, in any operations the annihilator can be replaced by this formula.

Example 4.1. Consider the affine matrix function $N(x)=\left(\begin{array}{ccc}x_{2} & -x_{1} & 0 \\ 2 x_{2}+x_{3} & 0 & 1-3 x_{1}\end{array}\right)$, where $x \in \mathbb{R}^{3}$. Its coefficient matrix looks like

$$
\Theta_{N}=\left(\begin{array}{cccc|cccc|cccc}
0 & 0 & 1 & 0 & 0 & -1 & 0 & 0 & 0 & 0 & 0 & 0 \\
0 & 0 & 2 & 1 & 0 & 0 & 0 & 0 & 1 & -3 & 0 & 0
\end{array}\right)
$$

The first column of blocks $\left(\begin{array}{llll}0 & 0 & 1 & 0 \\ 0 & 0 & 2 & 1\end{array}\right)$ of $\Theta_{N}$ corresponds to the first column of $N(x)$, since

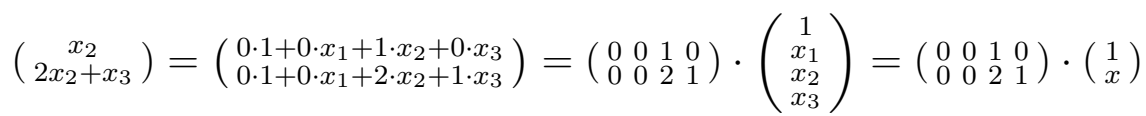

Therefore, knowing $\Theta_{N}$ one can uniquely reconstruct the original annihilator $N(\omega)$ in the following way:

$$
N(\omega)=\left(\begin{array}{cccc|cccc|cccc}
0 & 0 & 1 & 0 & 0 & -1 & 0 & 0 & 0 & 0 & 0 & 0 \\
0 & 0 & 2 & 1 & 0 & 0 & 0 & 0 & 1 & -3 & 0 & 0
\end{array}\right) \cdot\left(\begin{array}{cc}
1 & \\
x & \\
& 1 \\
& \\
& \\
x
\end{array}\right)=\Theta_{N} \cdot\left(I_{3} \otimes\left(\begin{array}{l}
1 \\
x
\end{array}\right)\right)
$$

Now we examine, how the structure of an annihilator $N(\omega)$ can be altered in order to reduce the dimension of the optimization problem. The next proposition will summarize the main result on choosing a smaller annihilator.

Proposition 4.2. Let $\Theta_{N}$ be the coefficient matrix of $N(\omega) \in \mathbb{R}^{q \times m}$. Assume that $\Theta_{N}$ is row rank deficient, i.e. $\operatorname{rank}\left(\Theta_{N}\right)=k<q$. Then, there exists an annihilator $\widehat{N}(\omega) \in \mathbb{R}^{k \times m}$ with $\widehat{\Theta}_{N}$ such that $\operatorname{rank}\left(\widehat{\Theta}_{N}\right)=k$ and $\mathfrak{F}_{\widehat{N}}=\mathfrak{F}_{N}$.

Proof. Without the loss of generality, we can assume that the first $k$ rows of $\Theta_{N}$ are linearly independent, therefore, $\Theta_{N}$ can be written in the form

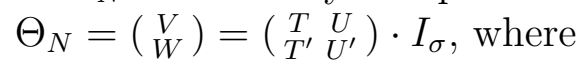

$$
\begin{array}{lll}
V \in \mathbb{R}^{k \times m(s+1)} & T \in \mathbb{R}^{k \times k} \text { (invertible) } & U \in \mathbb{R}^{k \times(m(s+1)-k)} \\
W \in \mathbb{R}^{(q-k) \times m(s+1)} & T^{\prime} \in \mathbb{R}^{(q-k) \times k} & U^{\prime} \in \mathbb{R}^{(q-k) \times(m(s+1)-k)}
\end{array}
$$

The elements of matrix $I_{\sigma} \in \mathbb{R}^{m(s+1) \times m(s+1)}$ are given as $\left(I_{\sigma}\right)_{i j}=\delta_{\sigma(i) j}$, where $\delta_{i j}$ is the Kronecker delta function. Furthermore, $\sigma$ is a permutation, 
in which the first $k$ values are the indices of the linearly independent columns of $\Theta_{N}$, and the further $m(s+1)-k$ values correspond to the indices of the $m(s+1)-k$ number of linearly dependent columns of matrix $\Theta_{N}$. The introduction of $I_{\sigma}$ is needed since the first $k$ columns of $\Theta_{N}$ are typically not linearly independent.

The rectangular matrix $V$ contains the $k$ linearly independent rows of $\Theta_{N}$, therefore, every further row of $\Theta_{N}$ collected in $W$ can be expressed as a linear combination of rows in $V$. This means that there exists a $(q-k) \times k$ matrix $\Gamma$ such that

$$
W=\Gamma V
$$

from which we get

$$
\Gamma=W V^{+} \in \mathbb{R}^{(q-k) \times k} .
$$

Since $V \in \mathbb{R}^{k \times m(s+1)}$ is full row-rank, the right pseudoinverse $V^{+}$of $V$ can be computed as

$$
V^{+}=V^{T}\left(V V^{T}\right)^{-1},
$$

where $V V^{+}=I$. Therefore, $\Gamma$ is uniquely defined. Consequently, there exists a matrix $S=\left(\begin{array}{cc}T^{-1} & 0 \\ -\Gamma & I\end{array}\right)$ and its inverse $S^{-1}=\left(\begin{array}{cc}T & 0 \\ \Gamma T & I\end{array}\right)$, such that

$\widehat{\Theta}_{N}:=S \cdot \Theta_{N}=\left(\begin{array}{cc}I & T^{-1} U \\ 0 & 0\end{array}\right) I_{\sigma} \Rightarrow N(\omega)=\Theta_{N} \cdot\left(I_{m} \otimes\left(\begin{array}{c}1 \\ \omega\end{array}\right)\right)=S^{-1} \widehat{\Theta}_{N} \cdot\left(I_{m} \otimes\left(\begin{array}{c}1 \\ \omega\end{array}\right)\right)$.

Matrix $\widehat{\Theta}_{N}$ constitutes the reduced row echelon form of $\Theta_{N}$. Considering $L=\left(\begin{array}{ll}L_{1} & L_{2}\end{array}\right) \in \mathbb{R}^{m \times q}$ as a block matrix, $L_{1} \in \mathbb{R}^{m \times k}$ and $L_{2} \in \mathbb{R}^{m \times(q-k)}$, we can give the following expression for term $L N(\omega)$ of LMI (7):

$$
\begin{aligned}
& L N(\omega)=\left(\begin{array}{ll}
L_{1} & L_{2}
\end{array}\right) S^{-1} \widehat{\Theta}_{N}\left(I_{m} \otimes\left(\begin{array}{l}
1 \\
\omega
\end{array}\right)\right) \\
& =\left(\begin{array}{ll}
L_{1} T+L_{2} \Gamma T & L_{2}
\end{array}\right)\left(\begin{array}{cc}
I & T^{-1} U \\
0 & 0
\end{array}\right) I_{\sigma}\left(I_{m} \otimes\left(\begin{array}{c}
1 \\
\omega
\end{array}\right)\right)=\widehat{L} \widehat{N}(\omega)
\end{aligned}
$$

where matrix $\widehat{L}$ and annihilator $\widehat{N}(\omega)$ are defined as follows:

$$
\begin{aligned}
& \widehat{L}:=L_{1} T+L_{2} \Gamma T \in \mathbb{R}^{m \times k} \\
& \widehat{N}(\omega):=\left(\begin{array}{ll}
I & T^{-1} U
\end{array}\right) I_{\sigma}\left(I_{m} \otimes\left(\begin{array}{c}
1 \\
\omega
\end{array}\right)\right)=\left(\begin{array}{ll}
T^{-1} & 0
\end{array}\right) N(\omega) \in \mathbb{R}^{k \times m}
\end{aligned}
$$

Equation (25a) implies that for every matrix $P \in \mathcal{S}^{m}$ and $L=\left(L_{1} L_{2}\right) \in$ $\mathbb{R}^{m \times q}$, which satisfy (7), there exists a matrix $\widehat{L}(25 \mathrm{~b})$ such that

$$
\mathcal{L}_{\widehat{N}}(P, \widehat{L})=\mathcal{L}_{N}(P, L) \succ 0, \forall \omega \in \Omega .
$$

This means that annihilators $N(\omega)$ and $\widehat{N}(\omega)$ generate the same feasible set for inequality (7). 
We note that the number of free decision variables collected in $\widehat{L}$ are less $(p \cdot k)$ than the number of variables in $L(p \cdot q)$. In other words, using annihilator $\widehat{N}$ instead of $N$ will lead us to a dimensionally reduced optimization problem, while the feasible set of the LMI constraint (7) remains the same.

For simplicity, we call an annihilator $N$ redundant if its coefficient matrix is row-rank deficient. Furthermore, let us call $\widehat{N}$ the row reduced f-equivalent of annihilator $N$ ( $f$-equivalent, in the sense that their generated feasible sets are the equal).

\subsection{Maximal annihilators}

In this subsection, we introduce the notion of maximal annihilators, which generate the largest possible feasible set for a fixed set of rational functions in $z(\omega)$.

It is obvious that an infinite number of annihilator rows $r(\omega)$ exists, such that $r(\omega) z(\omega)=0$. However, we have seen in the previous section that for any fixed rational function $z(\omega)$, there exists a maximal number for the rows of annihilators $q_{1} \leq m(s+1)$, to which if we append any further rows, the feasible set will not change anymore, since the coefficient matrix of the resulting annihilator will be row rank-deficient.

Lemma 4.3. For any two annihilators $N_{1}(\omega), N_{2}(\omega)$ of $z(\omega)$, we can construct an annihilator $N_{12}(\omega):=\left(\begin{array}{c}N_{1}(\omega) \\ N_{2}(\omega)\end{array}\right)$, such that $\mathfrak{F}_{N_{1}} \cup \mathfrak{F}_{N_{2}} \subseteq \mathfrak{F}_{N_{12}}$.

Proof of Lemma 4.3. If $\left(P, L_{1}\right)$ is a solution for $\mathcal{L}_{N_{1}}\left(P, L_{1}\right) \succ 0, \forall \omega \in \Omega$, meaning that $P \in \mathfrak{F}_{N_{1}}$, then there exists $L_{12}=\left[L_{1}, 0\right] \in \mathbb{R}^{m \times\left(q_{1}+q_{2}\right)}$ such that $\mathcal{L}_{N_{12}}\left(P, L_{12}\right)=\mathcal{L}_{N_{1}}\left(P, L_{1}\right) \succ 0, \forall \omega \in \Omega$. Consequently, $\mathfrak{F}_{N_{1}} \subseteq \mathfrak{F}_{N_{12}}$. In the same way, we can prove that $\mathfrak{F}_{N_{2}} \subseteq \mathfrak{F}_{N_{12}}$.

As a consequence, we can say that there exists (a non-unique) annihilator $N_{1}$, such that $\mathfrak{F}_{N_{1}}$ is a superset of the feasible sets corresponding to every possible affine annihilators of a given $z(\omega)$.

Definition 4.4. We call an affine matrix function $N_{1}: \mathbb{R}^{s} \rightarrow \mathbb{R}^{q_{1} \times m}$ a maximal annihilator of $z(\omega)$ if for every possible annihilator $N, \mathfrak{F}_{N} \subseteq \mathfrak{F}_{N_{1}}$.

\subsection{Maximal annihilator generation}

In this section, we introduce an efficient method to generate a maximal annihilator. By construction, we consider the most general form of a possible 
row of an affine annihilator. From now on, we consider only a single annihilator row, therefore, the first index $(l)$ will be suppressed from notations (18) or (19). We represent a general row of the desired annihilator in the following form:

$$
r(\omega ; \vartheta)=\left(\vartheta_{i 0}+\sum_{j=1}^{s} \vartheta_{i j} \omega_{j}\right)_{i=\overline{1, m}}=\left(\vartheta_{10}+\sum_{j=1}^{s} \vartheta_{1 j} \omega_{j} \ldots \vartheta_{m 0}+\sum_{j=1}^{s} \vartheta_{m j} \omega_{j}\right)
$$

We assume that the elements of $z(\omega)$ are rational functions in $\omega$ :

$$
z^{T}(\omega)=\left(\frac{u_{1}(\omega)}{v_{1}(\omega)} \quad \cdots \frac{u_{m}(\omega)}{v_{m}(\omega)}\right), \text { where } u_{j}, v_{j} \text { are polynomials in } \omega .
$$

We are looking for the possible values of $\vartheta \in \mathbb{R}^{1 \times m(s+1)}$ such that $r(\omega ; \vartheta) z(\omega)=$ 0 , for every $\omega \in \mathbb{R}^{s}$. Through some algebraic manipulation, we derive a system of linear equations, in which the unknown parameters are $\vartheta_{i j}$.

After the reduction of the fractions appearing in $r(\omega ; \vartheta) z(\omega)$ to have a common denominator, we cancel out the common terms from the numerator and denominator of the resulting quotient of polynomials. Consequently, we obtain an irreducible fraction where both the numerator and denominator are polynomials in $\omega$ whose greatest common divisor is 1 . Then we expand the expression of the numerator and finally, we collect the coefficients of the basis monomial terms in the numerator, which will have the form:

$$
\text { numerator: } \sum_{k=1}^{K} c_{k}(\vartheta) p_{k}(\omega), \text { with } p_{k}(\omega)=\prod_{j=1}^{s} \omega_{j}^{n_{j}}, \quad n_{j} \in \mathbb{N}
$$

where the $p_{k}(\omega)$ are distinct monomials with coefficients $c_{k}(\vartheta)$, furthermore, $c_{k}(\vartheta)$ are affine functions of variables $\vartheta_{i j}$. The numerator in Eq. (28) is zero for every $\omega \in \mathbb{R}^{s}$ if and only if the coefficients are zero, respectively, i.e.

$$
c_{k}(\vartheta)=0, \quad k=\overline{1, K}
$$

Due to the fact that $r(\omega ; \vartheta)$ is a linear expression in $\vartheta_{i j}$, Eq. (29) is a system of linear equations of the form $\mathbf{A} \vartheta^{T}=\mathbf{b}$ where $\mathbf{A} \in \mathbb{R}^{K \times m(s+1)}$ is a constant matrix and $\vartheta$ is the coefficient matrix of the affine annihilator row $r(\omega ; \vartheta)$. Moreover, $\mathbf{b}=0$, because the trivial solution $(\vartheta=0)$ always satisfies the equality $r(\omega ; \vartheta) z(\omega)=0$. This system of linear equations is typically underdetermined (i.e. $K<m(s+1)$ ), therefore, an infinite number of solutions 
exists. Without the loss of generality, we can assume that $\mathbf{A}$ is a full-rank matrix, otherwise, we eliminate the rows, which make it rank-deficient. In Section 4.1, we have already discussed that the coefficient matrix of the annihilator should be of full-row-rank (otherwise it is redundant), thus, we are interested only in the linearly independent solutions of this system of linear equations, that can be given by the basis vectors of the null space of matrix A:

$$
\operatorname{span}\left\langle\vartheta_{1}^{T}, \ldots, \vartheta_{m(s+1)-K}^{T}\right\rangle=\operatorname{Ker}(\mathbf{A})
$$

The following procedure describes the steps of annihilator generation:

\section{Procedure 1 Annihilator generation}

Input data: $\omega, z(\omega), s=\operatorname{dim}(\omega), m=\operatorname{dim}(z(\omega))$

$$
\begin{aligned}
& 1 \quad r(\omega ; \vartheta):=\left(\begin{array}{lll}
\vartheta_{10} & \ldots & \vartheta_{m 0}
\end{array}\right)+\omega^{T}\left(\begin{array}{cccc}
\vartheta_{11} & \ldots & \vartheta_{m 1} \\
\dddot{\vartheta} & \ldots & \cdots \\
\vartheta_{1 s} & \ldots & \vartheta_{m s}
\end{array}\right) \\
& 2 \text { [num,den] := numden }(r(\omega ; \vartheta), z(\omega)) \\
& 3 \text { largest_coeff }:=\max (\operatorname{abs}(\operatorname{coeffs}(\operatorname{den}))) \\
& 4 \text { vars }:=\left(\begin{array}{llll}
\vartheta_{10} & \vartheta_{11} \ldots \vartheta_{1 s}\left|\vartheta_{20} \ldots \vartheta_{2 s}\right| \ldots \mid \vartheta_{m 0} \ldots \vartheta_{m s}
\end{array}\right) \\
& 5 \text { coeffs_w }=\text { coeffs (num / largest_coeff, } \omega \text { ) } \\
& 6[\mathrm{~A}, \mathrm{~b}]:=\text { equationsToMatrix }\left(\operatorname{coeff} \mathrm{s}_{-} \mathrm{w}==0 \text {, vars }\right) \\
& 7 \Theta_{N}:=\operatorname{null}(\mathrm{A})^{T} \\
& 8 \quad N:=\Theta_{N} \cdot\left(I_{m} \otimes\left(\begin{array}{l}
1 \\
\omega
\end{array}\right)\right)
\end{aligned}
$$

In the first line of the algorithm, we construct a general parameterized annihilator row $r(\omega ; \vartheta)$. The values of $\vartheta_{i j}$ should be determined such that the value of the scalar object $r(\omega ; \vartheta) \cdot z(\omega)$ is zero for every $\omega \in \mathbb{R}^{s}$. This is a symbolic operation which was actually implemented using the Symbolic Math Toolbox of Matlab.

Command numden $(r(\omega ; \vartheta), z(\omega))$ in the second line, computes the numerator (num) and the denominator (den) of the irreducible form of the rational function $r(\omega ; \vartheta) \cdot z(\omega)$.

Command coeffs $(p)$ returns the coefficients of the polynomial $p$ with respect to all the indeterminates of $p$. If a second argument $(\omega)$ is given, coeffs $(p(\omega), \omega)$ returns the coefficients of the polynomial $p(\omega)$ with respect to $\omega$.

Due to numerical reasons, we divide the numerator of $r(\omega ; \vartheta) \cdot z(\omega)$ with the largest coefficient appearing in its denominator. Every coefficient of the 
resulting scaled numerator should be zero, which can be expressed as a linear equation system in the parameters $\vartheta_{i j}$.

Function equationsToMatrix (coeffs_w==0, vars) determines matrix $\mathbf{A}$ of the system of linear equations defined by coeffs_w==0. The order of the unknown variables are given by vars.

Finally, the basis vectors of the null space of $\mathbf{A}$ is computed, which constitutes the coefficient matrix of the annihilator. By construction, the obtained coefficient matrix is full-rank:

$$
\Theta_{N}=\left(\begin{array}{lll}
\vartheta_{1}^{T} & \ldots & \vartheta_{q}^{T}
\end{array}\right)^{T} \in \mathbb{R}^{q \times m(s+1)}, \quad \text { where } q:=m(s+1)-K,
$$

and the corresponding affine annihilator $N(\omega)$ is maximal, since we have taken into consideration every possible affine annihilator row (see, Eq. (26)). In other words, any other affine annihilator row $r(\omega)$ can be written in the form (26), and it satisfies $r(\omega) z(\omega)=0$. Therefore, the transpose of its

coefficient matrix $\vartheta=\Theta_{r}$ is a solution of $\mathbf{A} \vartheta^{T}=0$, i.e. it is an element of the null space of $\mathbf{A}$, and thus it can be expressed as the linear combination of the rows appearing in $\Theta_{N}$. To conclude, the obtained annihilator is a nonredundant maximal annihilator for a fixed set of rational functions collected in $z(\omega)$.

\section{Illustrative examples and results}

In this section, we illustrate the applicability of the approach presented above through different numerical examples. The results presented in this section were computed in the Matlab environment. For symbolic computations, we applied Matlab's built-in Symbolic Math Toolbox based on Mupad. For linear fractional transformations (LFTs), we used the Enhanced LFR-toolbox [19, 20]. To model and solve semidefinite optimization (SDP) problems, YALMIP [22] with Mosek solver [23] was used. The computations were processed on a desktop PC with Intel Core i5-4590 CPU at 3.30GHz and $16 \mathrm{~GB}$ of RAM.

\subsection{A third order rational system}

For comparative evaluation, first let us consider a third order rational system taken from [1]:

$$
\begin{aligned}
& \dot{x}_{1}=x_{2}+\varepsilon_{3} x_{3}+\varepsilon_{1} \zeta(x) \\
& \dot{x}_{2}=-x_{1}-x_{2}+\varepsilon_{2} x_{1}^{2} \\
& \dot{x}_{3}=\varepsilon_{3}\left(-2 x_{1}-2 x_{3}-x_{1}^{2}\right),
\end{aligned}
$$


where

$$
\zeta(x)=\frac{x_{1}}{x_{2}^{2}+1}, \varepsilon_{1}=\varepsilon_{2}=\varepsilon_{3}=\frac{1}{2}
$$

It is easy to see that (32) has an equilibrium point at $x^{*}=0$. This equilibrium is locally asymptotically stable, that can be justified by the negative real eigenvalues of the Jacobian matrix of the system at $x^{*}$. The problem to be solved is to compute a three-dimensional invariant domain as an estimate of the DOA around $x^{*}$.

Using the function sym2lfr of the LFR-toolbox, we obtain the following values for $A, B$ and $\pi$ :

$$
A=\left(\begin{array}{ccc}
0.5 & 1 & 0.5 \\
-1 & -1 & 0 \\
-1 & 0 & -1
\end{array}\right), B^{(0)}=\left(\begin{array}{cccc}
0 & 0 & -0.5 & 0 \\
0.5 & 0 & 0 & 0 \\
0 & -0.5 & 0 & 0
\end{array}\right), \pi^{(0)}=\left(\begin{array}{c}
x_{1}^{2} \\
x_{1}^{2} \\
x_{2}^{2} \zeta(x) \\
x_{2} \zeta(x)
\end{array}\right)
$$

We can see that monomial $x_{1}^{2}$ appears twice in $\pi$. Applying the automatic model simplification steps described in Section 3.1, we obtain the following reduced model.

$$
B=\left(\begin{array}{ccc}
0 & -0.5 & 0 \\
0.5 & 0 & 0 \\
-0.5 & 0 & 0
\end{array}\right), \text { with } \pi=\left(\begin{array}{c}
x_{1}^{2} \\
x_{2}^{2} \zeta(x) \\
x_{2} \zeta(x)
\end{array}\right)
$$

Using Procedure 1, we select a maximal annihilator for $\pi_{b}=\left(\begin{array}{l}x \\ \pi\end{array}\right)$ :

$$
N_{b}=\left(\begin{array}{cccccc}
x_{1} & 0 & 0 & -1 & 0 & 0 \\
x_{2} & 0 & 0 & 0 & -x_{2} & -1 \\
x_{3} & 0 & -x_{1} & 0 & 0 & 0 \\
0 & x_{1} & 0 & 0 & -x_{2} & -1 \\
\hline 0 & x_{3} & -x_{2} & 0 & 0 & 0 \\
0 & 0 & 0 & 0 & 1 & -x_{2}
\end{array}\right)=:\left(\begin{array}{c}
C_{b} \\
\aleph_{\pi_{b}}
\end{array}\right)
$$

If we decompose $N_{b}$ as shown above, we are able to give a possible annihilator $\widetilde{N}_{a}$ for $\pi_{a}$ as it is presented in [1, Eqs. $\left.(40,43)\right]$. Alternatively, we can generate a maximal annihilator $N_{a}$ as we have described in Procedure 1 . In this example, we used $\widetilde{N}_{a}$ in the computations.

As it was mentioned in Section 2, in order to obtain a larger estimate, the Lyapunov function is constrained to lie between the values $1 \leq V(x, \delta) \leq \tau_{k}$ on all facets $\mathcal{F}_{k}$ of the polytope $\mathcal{X}$, for $k=\overline{1, M_{\mathcal{X}}}$. Solving the LMIs (8), (10) and $[1$, Section 5, Eqs. (89) and (90)] for the polytope

$$
\mathcal{X}=[-4.87,4.58] \times[-5.95,6.29] \times[-10.04,8.71]
$$


an estimate is obtained, which is illustrated in Figure 2. Furthermore, some trajectories $x(t)$ are shown in Figure 3 from different initial conditions chosen from the inside of $\varepsilon_{1}$, typically, near the edges of $\varepsilon_{1}$. In the same figure, the value of the Lyapunov function $V$ and its time derivative $\dot{V}$ can be seen along the trajectories.

The volume of the estimated DOA for the system in [1] is $32.022 \mathrm{cu}-$ bic units $\left(u^{3}\right)$. Solving the optimization model constructed by our improved automated algorithm, the volume of the obtained invariant level set is approximately $717.92 u^{3}$. Polytope $\mathcal{X}$ was evaluated automatically using an iterative method presented in [24].

\subsection{D uncertain Lotka-Volterra system with an uncertain positive equilib- rium point.}

The system equation of an $n$-dimensional Lotka-Volterra (LV) model has the form

$$
\dot{\bar{x}}=\operatorname{diag}(\bar{x})(A \bar{x}+b), \quad A \in \mathbb{R}^{n \times n}, \quad b \in \mathbb{R}^{n},
$$

where $\operatorname{diag}(x), x \in \mathbb{R}^{n}$ denotes an $n \times n$ square diagonal matrix with $x_{1}, \ldots, x_{n}$ in the main diagonal. We translate the system into its (by assumption, unique) interior equilibrium point $\bar{x}^{*}=-A^{-1} b$ via introducing the centered state vector $x=\bar{x}-\bar{x}^{*}$. Then, we obtain an autonomous quasi-LPV system of the form $\dot{x}=\mathcal{A}(x) x$, where the matrix function $\mathcal{A}$ can be expressed as: $\mathcal{A}(x)=\operatorname{diag}\left(x+\bar{x}^{*}\right) A$.

Let us consider a 2-dimensional uncertain LV system, with an uncertain model matrix $A(\delta)$ and a constant vector $b$, as follows:

$$
A(\delta)=\left(\begin{array}{cc}
-\delta_{1} & -3 \\
1.4 & \delta_{2}
\end{array}\right), \quad b=\left(\begin{array}{c}
5 \\
-2.4
\end{array}\right), \quad \text { where } \begin{aligned}
& \delta_{1} \in[1.8,2.2] \\
& \delta_{2} \in[0.8,1.0]
\end{aligned}
$$

One can immediately notice that the equilibrium point depends on the actual values of the uncertain parameters. The nontrivial equilibrium point can be given as a function of $\delta$ :

$$
\bar{x}^{*}(\delta)=\zeta(\delta)\left(\begin{array}{c}
7.2-5 \delta_{2} \\
7-2.4 \delta_{1}
\end{array}\right), \quad \text { where } \zeta(\delta)=\frac{1}{4.2-\delta_{1} \delta_{2}}
$$

Since the value of $\delta_{1} \delta_{2}$ is between 1.44 and 2.2 , the equilibrium point $\bar{x}^{*}(\delta) \in \mathbb{R}^{n}$ is a smooth well-defined rational function of the uncertain parameters. The 


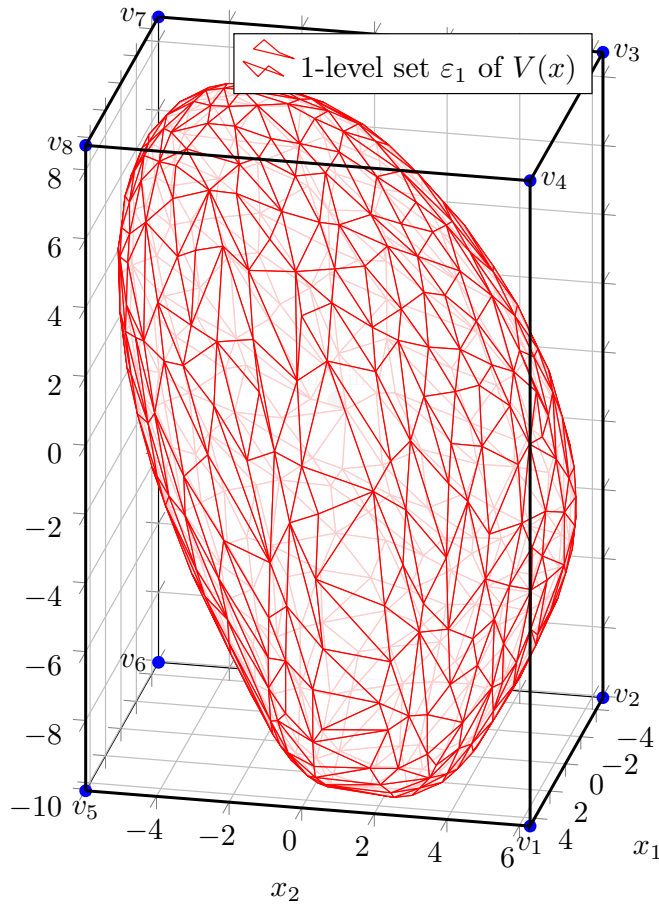

trajectories $x(t)$

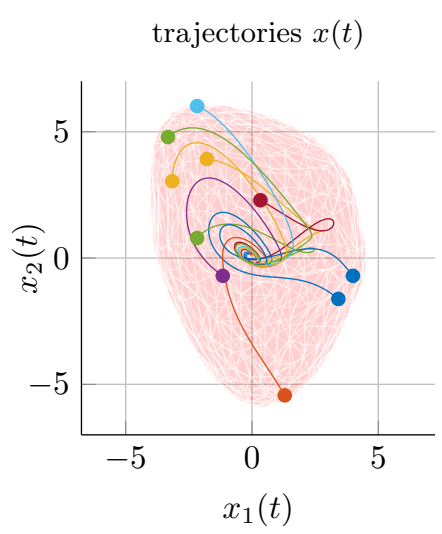

Figure 2: (left) The red volume illustrates the 1-level set $\varepsilon_{1}$ of the Lyapunov function obtained for the third order rational system presented in Section (5.1), the outer rectangular polygon having black edges constitutes polytope $\mathcal{X}$. The approximated volume of $\varepsilon_{1}$ is $717.92 u^{3}$, and the volume of $\mathcal{X}$ is 2168.59.

Figure 3: (below) The trajectories illustrate the solutions $x(t)$ of the third order rational system (32), with different initial conditions $x(0) \in \varepsilon_{1}$ (illustrated by colored dots). In the pair of figures on the right, we present the values of the Lyapunov function $V(t)=V(x(t))$ and its time derivative $\dot{V}(t)$ along the trajectories $x(t)$. We can observe that all trajectories converge to the origin, since $V(t)$ is strictly decreasing for every trajectory.
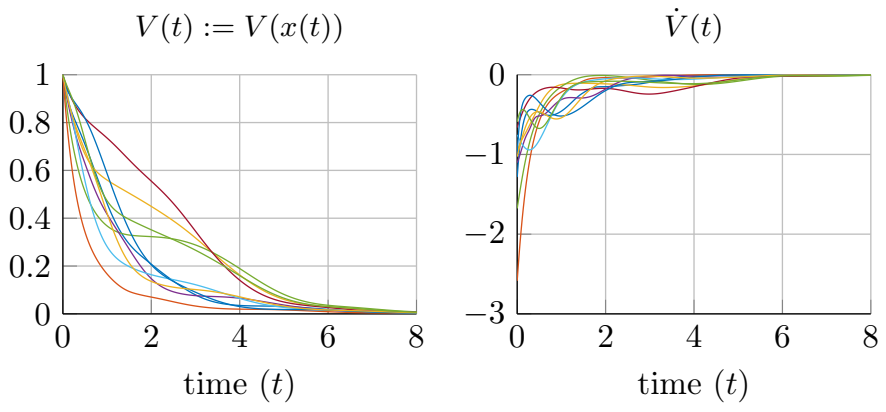
equation of the centered system is the following:

$\dot{x}=f(x, \delta)=\zeta(\delta)\left(\begin{array}{c}x_{1}^{2} \delta_{1}^{2} \delta_{2}-4.2 x_{1}^{2} \delta_{1}+3 x_{1} x_{2} \delta_{1} \delta_{2}-12.6 x_{1} x_{2}+5 x_{1} \delta_{1} \delta_{2}-7.2 x_{1} \delta_{1}+15 x_{2} \delta_{2}-21.6 x_{2} \\ -1.4 x_{1} x_{2} \delta_{1} \delta_{2}+5.88 x_{1} x_{2}-3.36 x_{1} \delta_{1}+9.8 x_{1}-1 x_{2}^{2} \delta_{1} \delta_{2}^{2}+4.2 x_{2}^{2} \delta_{2}-2.4 x_{2} \delta_{1} \delta_{2}+7 x_{2} \delta_{2}\end{array}\right)$

We can observe that the degrees of the monomial terms in the numerators of $f(x, \delta)$ are at most 2 with respect to the elements of $x$. Furthermore, the monomials contain at most third order terms of the uncertain parameters, and each uncertain parameter is at most on the 2nd power. Consequently, a reasonable set of rational functions to appear in the Lyapunov function is the following:

$$
\begin{aligned}
\varpi=\left\{p_{i}(x) q_{j}(\delta) \zeta(\delta) \mid\right. & p_{i}(x) \in\left\{x_{1}, x_{2}, x_{1}^{2}, x_{1} x_{2}, x_{2}^{2}\right\} \\
q_{j}(\delta) & \left.\in\left\{1, \delta_{1}, \delta_{2}, \delta_{1}^{2}, \delta_{1} \delta_{2}, \delta_{2}^{2}, \delta_{1}^{2} \delta_{2}, \delta_{1} \delta_{2}^{2}\right\}\right\},
\end{aligned}
$$

which constitutes $|\varpi|=40$ rational functions. In comparison, using the LFR-toolbox, we obtained a model for which the dimension of $\pi^{(0)}$ was $\operatorname{dim}\left(\pi^{(0)}\right)=28$. Finally, after the algebraic model simplification steps presented in Section 3.1, we obtain a set of rational functions $\pi$, which consists of only the following 14 functions:

$$
\begin{array}{r}
\pi=\zeta(\delta)\left(\begin{array}{lllllllll}
\delta_{1}^{2} \delta_{2} x_{1}^{2} & \delta_{1} x_{1} & \delta_{1} \delta_{2} x_{1} & \delta_{1} \delta_{2} x_{1} x_{2} & \delta_{1} \delta_{2} x_{2} & \delta_{1} \delta_{2}^{2} x_{2}^{2} & \delta_{1} \delta_{2} x_{1}^{2} & \delta_{2} x_{1} & \cdots \\
& \delta_{2} x_{2} & \delta_{2} x_{1} x_{2} & \delta_{2}^{2} x_{2}^{2} & \delta_{1} x_{1}^{2} & x_{1} x_{2} & \delta_{2} x_{2}^{2}
\end{array}\right)^{T}
\end{array}
$$

Then, Procedure 1 is applied to construct a maximal annihilator $N_{b}(x, \delta) \in$ $\mathbb{R}^{25 \times 16}$ for $\pi_{b}$. The annihilator $\widetilde{N}_{a}$ of $\pi_{a}$ is given as presented in [1, Eqs. $(40,43)]$.

The selected set of rational functions (39) generates a parameter dependent Lyapunov function $V(x, \delta)=\pi_{b}^{T} P \pi_{b}$. Since the steady state also depends on the uncertain parameter, we compute the Lyapunov function for (34) in the original coordinates system:

$$
\bar{V}(\bar{x}, \delta):=V\left(\bar{x}-\bar{x}^{*}(\delta), \delta\right)
$$

As it is described in [1, Eq. (95)], in case of a parameter dependent Lyapunov function, we can give a lower and an upper bound for $\bar{V}(\bar{x}, \delta)$ in the following way:

$$
\alpha_{1}(\bar{x}) \leq \bar{V}(\bar{x}, \delta) \leq \alpha_{2}(\bar{x}) \text { for all }(\bar{x}, \delta) \in \mathbb{R}^{n} \times \mathcal{D} \text { such that } \bar{x}-\bar{x}^{*}(\delta) \in \mathcal{X}
$$




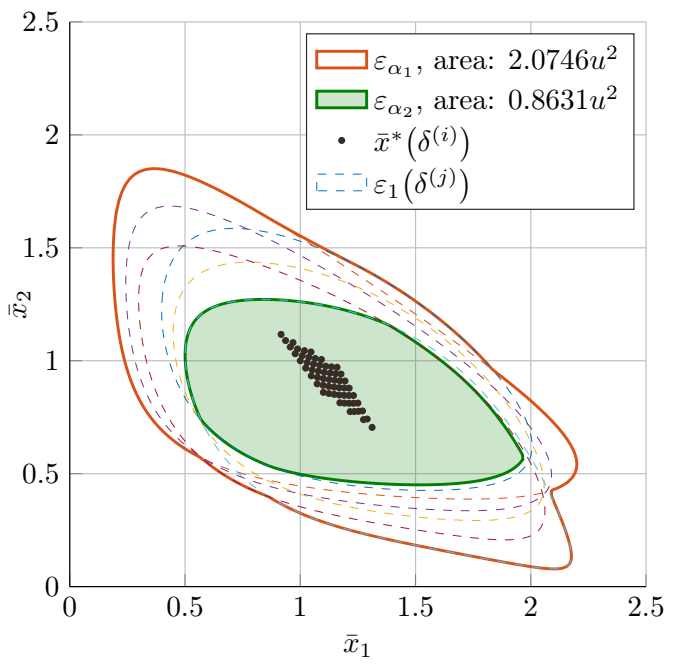

Figure 4: The estimated DOA for system (35) is given by two regions: $\varepsilon_{\alpha_{2}}$ bounded by the closed green line and $\varepsilon_{\alpha_{1}}$ bounded by the closed red line. The closed dashed lines illustrate the 1-level set of the Lyapunov function for some particular values of the uncertain parameters: $\delta^{(i)} \in\left\{\left(\delta_{1}, \delta_{2}\right) \mid \delta_{1} \in\{1.8,2,2.2\}, \delta_{2} \in\{0.8,1\}\right\}$. The black dots illustrate the equilibrium point of the system for certain values of $\delta$.

where $\alpha_{1}, \alpha_{2}: \mathbb{R}^{n} \rightarrow \mathbb{R}$ are scalar valued positive definite proper functions, thus their 1-level set $\varepsilon_{\alpha_{1}}=\left\{\bar{x} \in \mathbb{R}^{n} \mid \alpha_{1}(\bar{x}) \leq 1\right\}, \varepsilon_{\alpha_{2}}=\left\{\bar{x} \in \mathbb{R}^{n} \mid \alpha_{2}(\bar{x}) \leq 1\right\}$ are compact sets. These bounds should approach the Lyapunov function as much as possible, in order to obtain a larger DOA, which is described by the two level sets $\varepsilon_{\alpha_{2}} \subseteq \varepsilon_{\alpha_{1}}$. Even though $\varepsilon_{\alpha_{2}}$ is not invariant, we can state that any trajectory starting from an initial condition from the inside of $\varepsilon_{\alpha_{2}}$ will not leave $\varepsilon_{\alpha_{1}}$. Figure 4 illustrates the estimated DOA for system (35) in the original coordinates system.

Remark 5.1. Regions $\varepsilon_{\alpha_{1}}$ and $\varepsilon_{\alpha_{2}}$ can be numerically approximated by considering the union and the intersection, respectively, of the 1-level set of the Lyapunov function for discrete values of the uncertain parameters (e.g., one can consider a mesh grid on $\mathcal{D}$ ).

The polytope $\mathcal{X}$ was chosen manually to be

$$
\begin{aligned}
\mathcal{X}=\operatorname{co}\{ & (-0.584,-0.4651),(1.3487,-0.433),(0.6261,0.2426) \\
& (-0.1807,-0.7064),(-0.4412,0.8298),(-0.8782,0.7172) \\
& (1.0798,-0.7788),(1.3739,-0.5938),(-0.7941,-0.1354)\}
\end{aligned}
$$

where $\operatorname{co}(\cdot)$ denotes the convex hull of a given set. 


\subsection{A simple disease model}

In this example, we consider a susceptible-infectious-recovered-deceased (SIRD) model taken from [25]. The system equations are the following:

$$
\begin{aligned}
& \dot{\bar{x}}_{1}=\pi-\left(\beta_{1} \bar{x}_{2}+\beta_{2} \bar{x}_{4}+\lambda \bar{x}_{5}\right) \bar{x}_{1}-\mu \bar{x}_{1} \\
& \dot{\bar{x}}_{2}=\left(\beta_{1} \bar{x}_{2}+\beta_{2} \bar{x}_{4}+\lambda \bar{x}_{5}\right) \bar{x}_{1}-(\mu+\delta+\gamma) \bar{x}_{2} \\
& \dot{\bar{x}}_{3}=\gamma \bar{x}_{2}-\mu \bar{x}_{3} \\
& \dot{\bar{x}}_{4}=(\mu+\delta) \bar{x}_{2}-b \bar{x}_{4} \\
& \dot{\bar{x}}_{5}=\sigma+\xi \bar{x}_{2}+\alpha \bar{x}_{4}-\eta \bar{x}_{5}
\end{aligned}
$$

where $\bar{x}_{1}, \bar{x}_{2}, \bar{x}_{3}, \bar{x}_{4}$ denote the scaled numbers of susceptible, infectious, recovered and deceased human individuals, respectively, while $\bar{x}_{5}$ denotes the scaled concentration of virus pathogens in the environment. The values of model parameters are chosen to be as follows: $\pi=10, \eta=0.03, \xi=0.04, \alpha=$ $0.04, \delta=0.05, \beta_{1}=0.006, \beta_{2}=0.012, \lambda=0.01, \gamma=0.06, \mu=0.5, b=0.8$, $\sigma=0$. For this parameter configuration, system (43) has a unique positive (endemic) equilibrium point $\bar{x}^{*}=\left(\begin{array}{lllll}16.5986 & 2.788 & 0.3346 & 1.9168 & 6.273\end{array}\right)^{T}$, which is locally asymptotically stable [25, Theorem 5.1]. The value of $\bar{x}^{*}$ can be derived analytically, as it was shown in [25]. If we introduce the centered state variable $x=\bar{x}-\bar{x}^{*}$, the numerical form of the centered system's equation will be the following:

$$
\begin{aligned}
& \dot{x}_{1}=-0.006 x_{1} x_{2}-0.012 x_{1} x_{4}-0.01 x_{1} x_{5}-0.6025 x_{1}-0.0996 x_{2}-0.1992 x_{4}-0.166 x_{5} \\
& \dot{x}_{2}=0.006 x_{1} x_{2}+0.012 x_{1} x_{4}+0.01 x_{1} x_{5}+0.1025 x_{1}-0.5104 x_{2}+0.1992 x_{4}+0.166 x_{5} \\
& \dot{x}_{3}=0.06 x_{2}-0.5 x_{3} \\
& \dot{x}_{4}=0.55 x_{2}-0.8 x_{4} \\
& \dot{x}_{5}=0.04 x_{2}+0.04 x_{4}-0.03 x_{5}
\end{aligned}
$$

Considering every second order monomial of the state variables $x_{1}, x_{2}, x_{4}$ and $x_{5}$ to appear in $\pi$, we will have $\operatorname{dim}(\pi)=10$. If we skip every second order monomial in which a certain state variable is on the power of 2 (i.e., monomials of the form $x_{i}^{2}$ ), we obtain $\operatorname{dim}(\pi)=6$ monomials. Using the LFR-toolbox, we obtained the following model

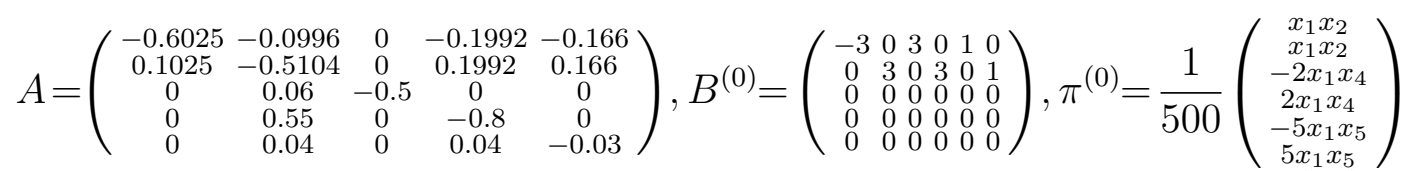



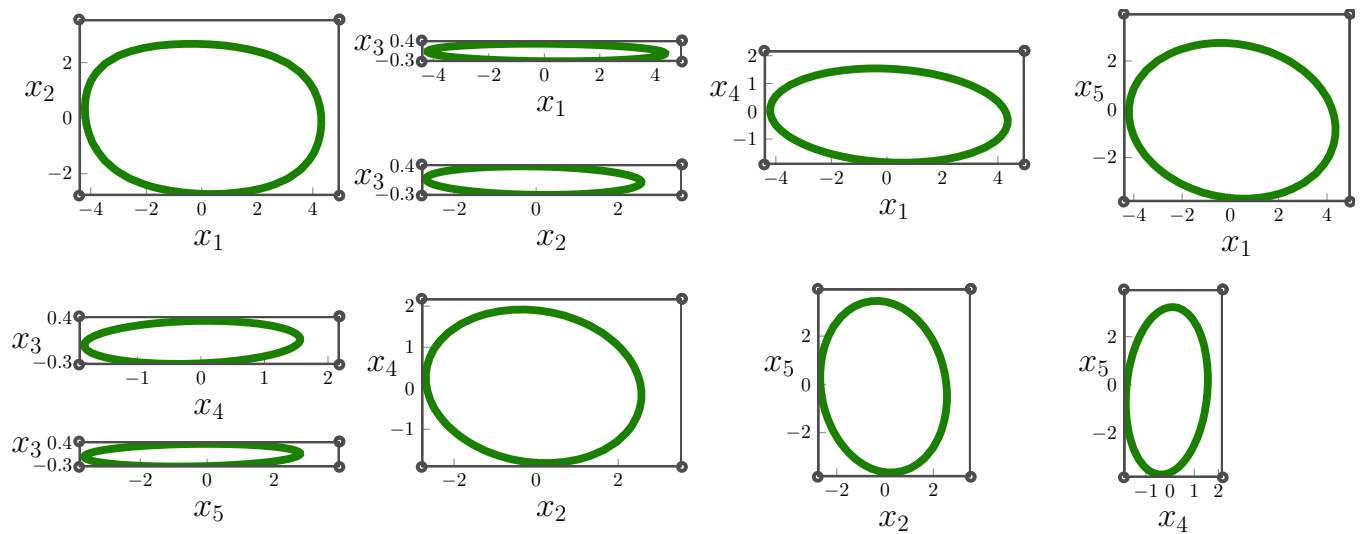

Figure 5: DOA for the 5-dimensional disease model. The area bounded by the closed green line illustrates the cross sections of the obtained invariant domain. The gray rectangle is the cross section of polytope $\mathcal{X}$ along the respective axes.

In comparison, after the proposed model simplification steps, we have a model, in which $\pi$ contains only three distinct monomials, which also appear in the system equation:

$$
B=\left(\begin{array}{ccc}
-0.006 & -0.012 & -0.01 \\
0.006 & 0.012 & 0.01 \\
0 & 0 & 0 \\
0 & 0 & 0 \\
0 & 0 & 0
\end{array}\right), \quad \pi=\left(\begin{array}{l}
x_{1} x_{2} \\
x_{1} x_{4} \\
x_{1} x_{5}
\end{array}\right)
$$

The annihilators for both $\pi_{a}$ and $\pi_{b}$ were selected using Procedure 1 . We used a rectangular polytope, which is evaluated iteratively as it is described in [24]. The final polytope is the following:

$$
\mathcal{X}=[-4.42,4.96] \times[-2.79,3.55] \times[-0.33,0.43] \times[-1.92,2.18] \times[-3.83,3.96] \text {. }
$$

The volume of the obtained invariant region is approximately $107.22 u^{5}$. In comparison, the volume of $\mathcal{X}$ is $1444.34 u^{5}$. Figure 5 illustrates the cross sections of the estimated DOA along the different axes.

\subsection{Comparative evaluation of the time-inverted Van der Pol system}

In this subsection, we estimate the domain of attraction of a benchmark model, the time-inverted Van der Pol system given by the equations

$$
\begin{aligned}
& \dot{x}_{1}=-x_{2} \\
& \dot{x}_{2}=x_{1}-\left(1-x_{1}^{2}\right) x_{2},
\end{aligned}
$$


for which the origin is a locally asymptotically stable equilibrium point.

The estimate is computed for different configurations. Firstly, we used the set of monomials proposed in [1, Example 7.1]:

$$
\pi^{(7)}=\left(\begin{array}{lllllll}
x_{1}^{2} & x_{1} x_{2} & x_{2}^{2} & x_{1}^{3} & x_{1}^{2} x_{2} & x_{1} x_{2}^{2} & x_{2}^{3}
\end{array}\right)^{T} .
$$

For this selection the structure of the time derivative of the Lyapunov function can be described by the polynomial terms of $\pi_{a}=\pi_{a}^{(7)}$ [1, Eq. (57)], which is given in AppendixA, Eq. (A.6). A possible selection for the annihilators of $\pi_{b}^{(7)}$ and $\pi_{a}^{(7)}$ are given by the formulas of $[1$, Eqs. $(28,34,40,43)]$ as

$$
\widetilde{N}_{b}^{(7)}=\left(\begin{array}{c}
C_{b}^{(7)} \\
\aleph_{\pi_{b}}^{(7)}
\end{array}\right), \quad \widetilde{N}_{a}^{(7)}=\left(\begin{array}{c}
C_{a}^{(7)} \\
\aleph_{\pi_{a}}^{(7)}
\end{array}\right) .
$$

According to Definition 4.4, $\widetilde{N}_{b}^{(7)}$ is a maximal annihilator of $\pi_{b}^{(7)}$, however, $\widetilde{N}_{a}^{(7)}$ is not a maximal annihilator of $\pi_{a}^{(7)}$. Using Procedure 1, we obtained a maximal annihilator for both $\pi_{b}^{(7)}$ and $\pi_{a}^{(7)}$. These are denoted by $N_{b}^{(7)}$ and $N_{a}^{(7)}$.

Secondly, we used the reduced set of monomials obtained through the method presented in Section 3, which is

$$
\pi^{(2)}=\left(\begin{array}{ll}
x_{1}^{2} x_{2} & x_{1} x_{2}
\end{array}\right)^{T} .
$$

Using Procedure 1, we obtained the following maximal annihilator for $\pi_{b}^{(2)}$ :

$$
N_{b}^{(2)}=\left(\begin{array}{cccc}
x_{2} & 0 & 0 & -1 \\
0 & x_{1} & 0 & -1 \\
0 & 0 & 1 & -x_{1}
\end{array}\right)=:\left(\begin{array}{l}
C_{b}^{(2)} \\
\aleph_{\pi_{b}}^{(2)}
\end{array}\right) \in \mathbb{R}^{3 \times 4}
$$

If we decompose $N_{b}^{(2)}$ into separate annihilators $C_{b}^{(2)}$ and $\aleph_{\pi_{b}}^{(2)}$ as shown in (53), we can use the formulas of $[1$, Eqs. $(40,43)]$ to obtain a possible (nonmaximal) annihilator for $\pi_{a}^{(2)}$ (Eq. (A.6)):

$$
\widetilde{N}_{a}^{(2)}=\left(\begin{array}{c}
C_{a}^{(2)} \\
\aleph_{\pi_{a}}^{(2)}
\end{array}\right) \in \mathbb{R}^{18 \times 14}
$$

Using Procedure 1, a maximal annihilator $N_{a}^{(2)}$ is generated for $\pi_{a}^{(2)}$, that is shown in (A.8). The dimension and the number of non-redundant rows of each annihilator are listed in Table 3. 
For both sets of monomials, we evaluated the optimization problem for different selection of annihilator pairs for $\pi_{b}^{(\cdot)}$ and $\pi_{a}^{(\cdot)}$ and for three different polytopes $\mathcal{X}_{0} \subset \mathcal{X}_{1} \subset \mathcal{X}_{2}$ (A.1). The processing time, the number of free decision variables, the dimensions of the optimization problem and the area of the obtained invariant region $\varepsilon_{1}$ for the different cases are listed in Table 1 and Table 2, in which the first three columns highlight the actual polytope and the selected annihilators for $\pi_{b}$ and $\pi_{a}$, respectively. The 4th column ("\#vars") contains the number of free scalar decision variables of the resulting optimization problem. The 6th column ("time") shows the measured processing time of the optimization solver. The last two columns show the approximated area of the obtained invariant region $\varepsilon_{1}$ and that of the polytope $\mathcal{X}$., respectively. Where no area information is available for $\varepsilon_{1}$, the optimization problem did not return successfully, i.e. the problem is infeasible or numerical issues occurred.

In case of $\pi^{(7)}$, the area of the obtained invariant region is practically the same for the different annihilator selections, and it is very close to the area of the true DOA. It is worth mentioning that the processing time is higher when $N_{a}^{(2)}$ is used instead of the one proposed in [1], since the dimension of $N_{a}^{(2)}$ is larger, and thus more decision variables are involved in the optimization problem. When using polytope $\mathcal{X}_{2}$, the solver did not return successfully due to numerical problems.

In the case of the reduced set of monomials $\pi^{(2)}$, the results confirm that the optimization problem using a maximal annihilator for $\pi_{a}^{(2)}$ is less conservative than the optimization problem when using annihilator $\tilde{N}_{a}^{(2)}$.

In all cases, the invariant level sets were enlarged in the framework of the optimization by using the objective function proposed in [1, Eq. (91)].

\section{Conclusions}

In this work, we presented an automatic rational function set selection algorithm based on the linear fractional transformation (LFT) and further systematic algebraic simplification steps. It was shown using different case studies that the proposed method generally uses less number of uncertain rational nonlinear function terms in the Lyapunov function computation problem compared to other known solutions in the literature. This results in the dimension reduction of the optimization problem corresponding to the DOA-estimation which is computationally clearly advantageous. At the 


\begin{tabular}{|c|c|c|c|c|c|c|c|}
\hline $\mathcal{X}$ & $N_{b}$ & $N_{a}$ & \#vars & number and size of LMIs & time & area $\left(\varepsilon_{1}\right)$ & area $(\mathcal{X})$ \\
\hline $\mathcal{X}_{0}$ & $\widetilde{N}_{b}^{(7)}$ & $\widetilde{N}_{a}^{(7)}$ & 5065 & $40 \cdot(9 \times 9)+8 \cdot(34 \times 34)$ & 17.91 & 11.263 & 11.6625 \\
$\mathcal{X}_{0}$ & $N_{b}^{(7)}$ & $\widetilde{N}_{a}^{(7)}$ & 4538 & $40 \cdot(9 \times 9)+8 \cdot(34 \times 34)$ & 16.19 & 11.253 & 11.6625 \\
$\mathcal{X}_{0}$ & $N_{b}^{(7)}$ & $N_{a}^{(7)}$ & 4878 & $40 \cdot(9 \times 9)+8 \cdot(34 \times 34)$ & 21.64 & 11.252 & 11.6625 \\
\hline $\mathcal{X}_{1}$ & $\widetilde{N}_{b}^{(7)}$ & $\widetilde{N}_{a}^{(7)}$ & 5065 & $40 \cdot(9 \times 9)+8 \cdot(34 \times 34)$ & 16.60 & 11.326 & 11.7328 \\
$\mathcal{X}_{1}$ & $N_{b}^{(7)}$ & $\widetilde{N}_{a}^{(7)}$ & 4538 & $40 \cdot(9 \times 9)+8 \cdot(34 \times 34)$ & 16.34 & 11.322 & 11.7328 \\
$\mathcal{X}_{1}$ & $N_{b}^{(7)}$ & $N_{a}^{(7)}$ & 4878 & $40 \cdot(9 \times 9)+8 \cdot(34 \times 34)$ & 21.43 & 11.319 & 11.7328 \\
\hline $\mathcal{X}_{2}$ & $\widetilde{N}_{b}^{(7)}$ & $\widetilde{N}_{a}^{(7)}$ & 7039 & $70 \cdot(9 \times 9)+14 \cdot(34 \times 34)$ & 5.72 & - & 12.0465 \\
$\mathcal{X}_{2}$ & $N_{b}^{(7)}$ & $\widetilde{N}_{a}^{(7)}$ & 6188 & $70 \cdot(9 \times 9)+14 \cdot(34 \times 34)$ & 16.18 & - & 12.0465 \\
$\mathcal{X}_{2}$ & $N_{b}^{(7)}$ & $N_{a}^{(7)}$ & 6528 & $70 \cdot(9 \times 9)+14 \cdot(34 \times 34)$ & 21.85 & - & 12.0465 \\
\hline
\end{tabular}

Table 1: Numerical results for the model representation (3) where the set of monomials is chosen to be $\pi=\pi^{(7)}$. In each block (corresponding to the three different polytopes $\left.\mathcal{X}_{0} \subset \mathcal{X}_{1} \subset \mathcal{X}_{2}\right)$ the first row contains the results when the annihilators of $\pi_{b}^{(7)}$ and $\pi_{a}^{(7)}$ are chosen as proposed in [1], the third row illustrates the results, when we use the maximal non-redundant annihilator generated by Procedure 1 for both functions $\pi_{b}^{(7)}$ and $\pi_{a}^{(7)}$, the second row presents the results for the annihilator of $\pi_{a}^{(7)}$ proposed in [1] and for a non-redundant annihilator of $\pi_{b}^{(7)}$ generated by Procedure 1 .

\begin{tabular}{|c|c|c|c|c|c|c|c|}
\hline $\mathcal{X}$ & $N_{b}$ & $N_{a}$ & $\#$ vars & number and size of LMIs & time & area $\left(\varepsilon_{1}\right)$ & area $(\mathcal{X})$ \\
\hline $\mathcal{X}_{0}$ & $N_{b}^{(2)}$ & $\widetilde{N}_{a}^{(2)}$ & 634 & $40 \cdot(4 \times 4)+8 \cdot(14 \times 14)$ & 0.64 & 10.999 & 11.6625 \\
$\mathcal{X}_{0}$ & $N_{b}^{(2)}$ & $N_{a}^{(2)}$ & 704 & $40 \cdot(4 \times 4)+8 \cdot(14 \times 14)$ & 0.63 & 11.027 & 11.6625 \\
\hline $\mathcal{X}_{1}$ & $N_{b}^{(2)}$ & $\widetilde{N}_{a}^{(2)}$ & 634 & $40 \cdot(4 \times 4)+8 \cdot(14 \times 14)$ & 0.87 & - & 11.7328 \\
$\mathcal{X}_{1}$ & $N_{b}^{(2)}$ & $N_{a}^{(2)}$ & 704 & $40 \cdot(4 \times 4)+8 \cdot(14 \times 14)$ & 0.77 & 11.106 & 11.7328 \\
\hline $\mathcal{X}_{2}$ & $N_{b}^{(2)}$ & $\widetilde{N}_{a}^{(2)}$ & 904 & $70 \cdot(4 \times 4)+14 \cdot(14 \times 14)$ & 1.10 & - & 12.0465 \\
$\mathcal{X}_{2}$ & $N_{b}^{(2)}$ & $N_{a}^{(2)}$ & 974 & $70 \cdot(4 \times 4)+14 \cdot(14 \times 14)$ & 1.32 & 11.399 & 12.0465 \\
\hline
\end{tabular}

Table 2: Numerical results for the model representation (3) where the set of monomials is chosen as $\pi=\pi^{(2)}$. In each block the first row contains the results when the annihilator of $\pi_{a}^{(a)}$ is the one proposed in [1], while the annihilator of $\pi_{b}^{(2)}$ is generated by Procedure 1. The second row illustrates the results, when we use the maximal annihilator generated by Procedure 1 for both functions $\pi_{b}^{(2)}$ and $\pi_{a}^{(2)}$.

\begin{tabular}{|l|c|c|c||c|c|c|c|}
\hline$N^{(7)}$ & $\operatorname{dim}$ & \#n.r. & Eq. & $N^{(2)}$ & $\operatorname{dim}$ & \#n.r. & Eq. \\
\hline$\widetilde{N}_{b}^{(7)}$ & $16 \times 9$ & 13 & $(\mathrm{~A} .4)$ & - & - & - & - \\
$N_{b}^{(7)}$ & $13 \times 9$ & 13 & $(\mathrm{~A} .5)$ & $N_{b}^{(2)}$ & $3 \times 4$ & 3 & $(53)$ \\
$\widetilde{N}_{a}^{(7)}$ & $66 \times 34$ & 55 & $(\mathrm{~A} .9)$ & $\widetilde{N}_{a}^{(2)}$ & $18 \times 14$ & 15 & $(\mathrm{~A} .7)$ \\
$N_{a}^{(7)}$ & $74 \times 34$ & 74 & $(\mathrm{~A} .10)$ & $N_{a}^{(2)}$ & $23 \times 14$ & 23 & $(\mathrm{~A} .8)$ \\
\hline
\end{tabular}

Table 3: Size of annihilators (2nd and 6th columns), and the number of their nonredundant rows (3rd and 7th columns). The formula for each annihilator can be found in AppendixA. 
same time, the results show that the obtained set of rational functions is sufficiently representative considering the nonlinear terms of the system equation. Additionally, we have introduced an automatic method for affine annihilator computation. We have shown that the obtained annihilator is maximal in the sense that no other annihilator can result in a strictly larger solution set for the LMIs ensuring the Lyapunov conditions. The operation of the approach was illustrated through examples taken from the literature that confirmed the applicability of the proposed method.

\section{Acknowledgements}

The research was partially supported by the grant K115694 of the National Research, Development and Innovation Office - NKFIH. The project has also been supported by the European Union, co-financed by the European Social Fund through the grant EFOP-3.6.3-VEKOP-16-2017-00002. The second author is the grantee of the Bolyai János Scholarship of the Hungarian Academy of Sciences. The authors thank Dr. Balázs Á. Kulcsár for his suggestions. The authors are also grateful to the anonymous reviewers for their constructive comments. 


\section{References}

[1] A. Trofino, T. J. M. Dezuo, LMI stability conditions for uncertain rational nonlinear systems, International Journal of Robust and Nonlinear Control 24 (18) (2013) 3124-3169. doi:10.1002/rnc.3047.

[2] G. Chesi, Domain of attraction: analysis and control via SOS programming, Vol. 415, Springer Science \& Business Media, 2011. doi: 10.1007/978-0-85729-959-8.

[3] V. I. Zubov, L. F. Boron, Methods of AM Lyapunov and their Application, Noordhoff Groningen, 1964.

[4] F. Camilli, L. Grüne, F. Wirth, A generalization of Zubov's method to perturbed systems, SIAM Journal on Control and Optimization 40 (2) (2001) 496-515. doi:10.1137/S036301299936316X.

[5] A. Vannelli, M. Vidyasagar, Maximal Lyapunov functions and domains of attraction for autonomous nonlinear systems, Automatica 21 (1) (1985) 69-80. doi:10.1016/0005-1098 (85) 90099-8.

[6] Y. Ohta, H. Imanishi, L. Gong, H. Haneda, Computer generated Lyapunov functions for a class of nonlinear systems, IEEE Transactions on Circuits and Systems 40 (1993) 343-354. doi:10.1109/SSST.1989. 72503.

[7] P. Giesl, S. Hafstein, Construction of Lyapunov functions for nonlinear planar systems by linear programming, Journal of Mathematical Analysis and Applications 388 (2012) 463-479. doi:10.1016/j.jmaa.2011. 10.047 .

[8] S. Rozgonyi, K. M. Hangos, G. Szederkényi, Determining the domain of attraction of hybrid non-linear systems using maximal Lyapunov functions, Kybernetika 46 (2010) 19-37. doi:10338.dmlcz/140048.

[9] S. Boyd, L. El Ghaoui, E. Feron, V. Balakrishnan, Linear Matrix Inequalities in System and Control Theory, Vol. 15 of Studies in Applied Mathematics, SIAM, Philadelphia, PA, 1994.

[10] S. Boyd, L. Vandenberghe, Convex Optimization, Cambridge University Press, New York, NY, USA, 2004. 
[11] L. E. Ghaoui, G. Scorletti, Control of rational systems using linearfractional representations and linear matrix inequalities, Automatica 32 (9) (1996) 1273 - 1284. doi:10.1016/0005-1098(96)00071-4.

[12] U. Topcu, A. K. Packard, P. Seiler, Local stability analysis using simulations and sum-of-squares programming, Automatica 44 (10) (2008) 2669-2675. doi:10.1016/j.automatica.2008.03.010.

[13] D. Nesic, Extremum seeking control: convergence analysis, European Journal of Control 3-4 (2009) 331-347. doi:10.3166/ejc.15.331-347.

[14] I. Sarras, H. B. Siguerdidjane, R. Ortega, Stabilization of the experimental cart-pendulum system with proven domain of attraction, European Journal of Control 4 (2010) 329-340. doi:10.3166/ejc.16.329-340.

[15] L. F. Buzachero, E. Assuncao, E. R. Da Silva, M. C. Teixeira, New techniques for optimizing the norm of robust controllers of polytopic uncertain linear systems, INTECH Open Access Publisher, 2012. doi: $10.5772 / 38920$.

[16] S. Moheimani (Ed.), Perspectives in Robust Control, Vol. 268, SpringerVerlag New York, 2001. doi:10.1007/BFb0110609.

[17] C. W. Scherer, S. Weiland, Linear matrix inequalities in control, Lecture Notes, Dutch Institute for Systems and Control, Delft, The Netherlands.

[18] K. Zhou, J. C. Doyle, K. Glover, et al., Robust and optimal control, Vol. 40, Prentice hall New Jersey, 1996.

[19] S. Hecker, A. Varga, J.-F. Magni, Enhanced LFR-toolbox for MATLAB, 2004, pp. 25-29. doi:10.1109/CACSD. 2004.1393845.

[20] J.-F. Magni, Linear fractional representation toolbox (version 2.0) for use with MATLAB, Free Web publication http://www. cert. $\mathrm{fr} / \mathrm{dcsd} /$ idco/perso/Magni.

[21] P. Lambrechts, J. Terlouw, S. Bennani, M. Steinbuch, Parametric uncertainty modeling using LFTs, 1993, pp. 267-272.

[22] J. Löfberg, Yalmip : A toolbox for modeling and optimization in MATLAB, in: Proceedings of the CACSD Conference, Taipei, Taiwan, 2004. 
doi:10.1109/CACSD . 2004.1393890.

URL http://users.isy.liu.se/johanl/yalmip

[23] MOSEK ApS, The MOSEK optimization toolbox for MATLAB manual. Version 7.1 (Revision 28). (2015).

URL http://docs.mosek.com

[24] P. Polcz, G. Szederkényi, K. M. Hangos, Computational stability analysis of an uncertain bioreactor model, in: 13th International Symposium on Stability, Vibration, and Control of Machines and Structures - SVCS 2016, June 16-18, Budapest, Hungary, 2016, pp. 1-12.

[25] T. Berge, J.-S. Lubuma, G. Moremedi, N. Morris, R. Kondera-Shava, A simple mathematical model for Ebola in Africa, Journal of Biological Dynamics 11 (1) (2017) 42-74. doi:10.1080/17513758.2016.1229817.

[26] Graziano Chesi. Rational Lyapunov functions for estimating and controlling the robust domain of attraction. Automatica, 49(4):1051-1057, 2013. 


\section{AppendixA. Numerical results illustrated on the time-inverted Van der Pol system}

In this section, the numerical values are given for the objects mentioned in Section 5.4. The three polytopes $\mathcal{X}_{0} \subset \mathcal{X}_{1} \subset \mathcal{X}_{2}$ are

$$
\begin{array}{r}
\mathcal{X}_{0}=\operatorname{co}\{(1.788,-0.264),(-1.788,0.264),(1.201,2.374),(-1.201,-2.374) \\
(1.788,0.908),(-1.788,-0.908),(0.322,2.374),(-0.322,-2.374)\}, \\
\operatorname{area}\left(\mathcal{X}_{0}\right)=11.6625 \\
\mathcal{X}_{1}=\operatorname{co}\{(1.793,-0.265),(-1.793,0.265),(1.205,2.381),(-1.205,-2.381) \\
\quad(1.793,0.911),(-1.793,-0.911),(0.323,2.381),(-0.323,-2.381)\}, \\
\operatorname{area}\left(\mathcal{X}_{1}\right)=11.7328 \\
\mathcal{X}_{2}=\operatorname{co}\{(1.793,-0.265),(-1.793,0.265),(0.323,2.381),(-0.323,-2.381) \\
(1.793,0.911),(-1.793,-0.911),(0.675,2.493),(-0.675,-2.493) \\
(1.205,2.381),(-1.205,-2.381),(1.613,1.586),(-1.613,-1.586) \\
(1.864,0.275),(-1.864,-0.275)\} .
\end{array}
$$

$\operatorname{area}\left(\mathcal{X}_{2}\right)=12.0465$

where $\operatorname{co}(\cdot)$ denotes the convex hull of a given set. The two different sets of monomials to appear in the Lyapunov function are:

$$
\pi^{(7)}=\left(\begin{array}{lllllll}
x_{1}^{2} & x_{1} x_{2} & x_{2}^{2} & x_{1}^{3} & x_{1}^{2} x_{2} & x_{1} x_{2}^{2} & x_{2}^{3}
\end{array}\right)^{T}, \pi^{(2)}=\left(\begin{array}{lll}
x_{1}^{2} x_{2} & x_{1} x_{2}
\end{array}\right)^{T}
$$

Using Procedure 1, the maximal annihilator for $\pi_{b}^{(2)}$ is

$$
N_{b}^{(2)}=\left(\begin{array}{cccc}
x_{2} & 0 & 0 & -1 \\
0 & x_{1} & 0 & -1 \\
0 & 0 & 1 & -x_{1}
\end{array}\right)=:\left(\begin{array}{l}
C_{b}^{(2)} \\
\aleph_{\pi_{b}}^{(2)}
\end{array}\right) \in \mathbb{R}^{3 \times 4}
$$


According to $[1$, Eqs. $(28,34)]$, an annihilator of $\pi_{b}^{(7)}$ is $\widetilde{N}_{b}^{(7)}=\left(\begin{array}{l}C_{b}^{(7)} \\ \aleph_{\pi_{b}}^{(7)}\end{array}\right)$

$$
\widetilde{N}_{b}^{(7)}=\left(\begin{array}{ccccccccc}
x_{1} & 0 & -1 & 0 & 0 & 0 & 0 & 0 & 0 \\
x_{2} & 0 & 0 & -1 & 0 & 0 & 0 & 0 & 0 \\
0 & x_{2} & 0 & 0 & -1 & 0 & 0 & 0 & 0 \\
0 & 0 & x_{1} & 0 & 0 & -1 & 0 & 0 & 0 \\
0 & 0 & x_{2} & 0 & 0 & 0 & -1 & 0 & 0 \\
0 & 0 & 0 & x_{2} & 0 & 0 & 0 & -1 & 0 \\
0 & 0 & 0 & 0 & x_{2} & 0 & 0 & 0 & -1 \\
\hline x_{2} & -x_{1} & 0 & 0 & 0 & 0 & 0 & 0 & 0 \\
0 & 0 & x_{2} & -x_{1} & 0 & 0 & 0 & 0 & 0 \\
0 & 0 & 0 & x_{2} & -x_{1} & 0 & 0 & 0 & 0 \\
0 & 0 & 0 & 0 & 0 & x_{2} & -x_{1} & 0 & 0 \\
0 & 0 & 0 & 0 & 0 & 0 & x_{2} & -x_{1} & 0 \\
0 & 0 & 0 & 0 & 0 & 0 & 0 & x_{2} & -x_{1} \\
0 & x_{1} & 0 & -1 & 0 & 0 & 0 & 0 & 0 \\
0 & 0 & 0 & x_{1} & 0 & 0 & -1 & 0 & 0 \\
0 & 0 & 0 & 0 & x_{1} & 0 & 0 & -1 & 0
\end{array}\right) \in \mathbb{R}^{(7+9) \times 9}
$$

while, the maximal non-redundant annihilator for $\pi_{b}^{(7)}$ is

$$
N_{b}^{(7)}=\left(\begin{array}{ccccccccc}
x_{1} & 0 & -1 & 0 & 0 & 0 & 0 & 0 & 0 \\
x_{2} & 0 & 0 & -1 & 0 & 0 & 0 & 0 & 0 \\
0 & x_{1} & 0 & -1 & 0 & 0 & 0 & 0 & 0 \\
0 & x_{2} & 0 & 0 & -1 & 0 & 0 & 0 & 0 \\
0 & 0 & x_{1} & 0 & 0 & -1 & 0 & 0 & 0 \\
0 & 0 & x_{2} & 0 & 0 & 0 & -1 & 0 & 0 \\
0 & 0 & 0 & x_{1} & 0 & 0 & -1 & 0 & 0 \\
0 & 0 & 0 & x_{2} & 0 & 0 & 0 & -1 & 0 \\
0 & 0 & 0 & 0 & x_{1} & 0 & 0 & -1 & 0 \\
0 & 0 & 0 & 0 & x_{2} & 0 & 0 & 0 & -1 \\
0 & 0 & 0 & 0 & 0 & x_{2} & -x_{1} & 0 & 0 \\
0 & 0 & 0 & 0 & 0 & 0 & x_{2} & -x_{1} & 0 \\
0 & 0 & 0 & 0 & 0 & 0 & 0 & x_{2} & -x_{1}
\end{array}\right) \in \mathbb{R}^{13 \times 9}
$$

For both sets of monomials $\pi^{(7)}$ and $\pi^{(2)}$, we computed $\pi_{a}^{(7)}$ and $\pi_{a}^{(2)}$, respectively, which represent the structure of the time derivative of the Lya- 
punov function. The formula for $\pi_{a}^{(\cdot)}$ is given in [1, Eq. (57)].

$$
\pi_{a}^{(7)}=\left(\begin{array}{c}
\pi_{b}^{(7)} \\
-2 x_{1} x_{2} \\
x_{1}\left(x_{2} x_{1}^{2}+x_{1}-x_{2}\right)-x_{2}^{2} \\
2 x_{2}\left(x_{2} x_{1}^{2}+x_{1}-x_{2}\right) \\
-3 x_{1}^{2} x_{2} \\
x_{1}^{2}\left(x_{2} x_{1}^{2}+x_{1}-x_{2}\right)-2 x_{1} x_{2}^{2} \\
2 x_{1} x_{2}\left(x_{2} x_{1}^{2}+x_{1}-x_{2}\right)-x_{2}^{3} \\
3 x_{2}^{2}\left(x_{2} x_{1}^{2}+x_{1}-x_{2}\right) \\
-x_{1} x_{2} \\
-x_{2}^{2} \\
x_{1}\left(x_{2} x_{1}^{2}+x_{1}-x_{2}\right) \\
x_{2}\left(x_{2} x_{1}^{2}+x_{1}-x_{2}\right) \\
-x_{1}^{2} x_{2} \\
-x_{1} x_{2}^{2} \\
-x_{2}^{3} \\
-x_{1}^{3} x_{2} \\
-x_{1}^{2} x_{2}^{2} \\
-x_{1} x_{2}^{3} \\
-x_{2}^{4} \\
x_{1}^{2}\left(x_{2} x_{1}^{2}+x_{1}-x_{2}\right) \\
x_{1} x_{2}\left(x_{2} x_{1}^{2}+x_{1}-x_{2}\right) \\
x_{2}^{2}\left(x_{2} x_{1}^{2}+x_{1}-x_{2}\right) \\
x_{1}^{3}\left(x_{2} x_{1}^{2}+x_{1}-x_{2}\right) \\
x_{1}^{2} x_{2}\left(x_{2} x_{1}^{2}+x_{1}-x_{2}\right) \\
x_{1} x_{2}^{2}\left(x_{2} x_{1}^{2}+x_{1}-x_{2}\right) \\
x_{2}^{3}\left(x_{2} x_{1}^{2}+x_{1}-x_{2}\right)
\end{array}\right) \quad \pi_{a}^{(2)}=\left(\begin{array}{c}
\pi_{b}^{(2)} \\
-x_{1}^{2}\left(x_{2}-x_{1}\left(x_{1} x_{2}+1\right)\right)-2 x_{1} x_{2}^{2} \\
-x_{1}\left(x_{2}-x_{1}\left(x_{1} x_{2}+1\right)\right)-x_{2}^{2} \\
-x_{1} x_{2} \\
-x_{2}^{2} \\
-x_{1}\left(x_{2}-x_{1}\left(x_{1} x_{2}+1\right)\right) \\
-x_{2}\left(x_{2}-x_{1}\left(x_{1} x_{2}+1\right)\right) \\
-x_{1}^{2} x_{2}^{2} \\
-x_{1} x_{2}^{2} \\
-x_{1}^{2} x_{2}\left(x_{2}-x_{1}\left(x_{1} x_{2}+1\right)\right) \\
-x_{1} x_{2}\left(x_{2}-x_{1}\left(x_{1} x_{2}+1\right)\right) \\
\end{array}\right)
$$

According to $[1$, Eqs. $(40,43)]$, an annihilator for $\pi_{a}^{(2)}$ is $\widetilde{N}_{a}^{(2)}=\left(\begin{array}{l}C_{a}^{(2)} \\ \aleph_{\pi_{b}}^{(7)}\end{array}\right)$

$$
\widetilde{N}_{a}^{(2)}=\left(\begin{array}{cccccccccccccc}
x_{2} & 0 & 0 & -1 & 0 & 0 & 0 & 0 & 0 & 0 & 0 & 0 & 0 & 0 \\
0 & x_{1} & 0 & -1 & 0 & 0 & 0 & 0 & 0 & 0 & 0 & 0 & 0 & 0 \\
x_{1} & -x_{1}-x_{2} & x_{1} & 0 & 0 & -1 & 0 & 0 & 0 & 0 & 0 & 0 & 0 & 0 \\
x_{1} & -x_{1}-x_{2} & x_{1} & 0 & 0 & -1 & 0 & 0 & 0 & 0 & 0 & 0 & 0 & 0 \\
0 & -x_{1} & 0 & 0 & 0 & 0 & 1 & 0 & 0 & 0 & 0 & 0 & 0 & 0 \\
0 & -x_{2} & 0 & 0 & 0 & 0 & 0 & 1 & 0 & 0 & 0 & 0 & 0 & 0 \\
x_{1} & -x_{1} & x_{1} & 0 & 0 & 0 & 0 & 0 & 1 & 0 & 0 & 0 & 0 & 0 \\
x_{2} & -x_{2} & x_{2} & 0 & 0 & 0 & 0 & 0 & 0 & 1 & 0 & 0 & 0 & 0 \\
0 & 0 & 0 & 0 & 0 & 0 & -x_{2} & 0 & 0 & 0 & 0 & -1 & 0 & 0 \\
0 & 0 & 0 & 0 & 0 & 0 & 0 & -x_{1} & 0 & 0 & 0 & -1 & 0 & 0 \\
0 & 0 & 0 & 0 & 0 & 0 & 0 & 0 & -x_{2} & 0 & 0 & 0 & 0 & -1 \\
0 & 0 & 0 & 0 & 0 & 0 & 0 & 0 & 0 & -x_{1} & 0 & 0 & 0 & -1 \\
\hline 0 & 0 & 1 & -x_{1} & 0 & 0 & 0 & 0 & 0 & 0 & 0 & 0 & 0 & 0 \\
0 & 0 & 0 & 0 & 1 & -x_{1} & 0 & 0 & 0 & 0 & 0 & -1 & 0 & 0 \\
0 & 0 & 0 & 0 & 0 & 0 & x_{2} & -x_{1} & 0 & 0 & 0 & 0 & 0 & 0 \\
0 & 0 & 0 & 0 & 0 & 0 & 0 & 0 & x_{2} & -x_{1} & 0 & 0 & 0 & 0 \\
0 & 0 & 0 & 0 & 0 & 0 & 0 & 0 & 0 & 0 & 1 & -x_{1} & 0 & 0 \\
0 & 0 & 0 & 0 & 0 & 0 & 0 & 0 & 0 & 0 & 0 & 0 & 1 & -x_{1}
\end{array}\right) \in \mathbb{R}^{(12+6) \times 14}
$$


The maximal annihilator for $\pi_{a}^{(2)}$ is

$$
N_{a}=\left(\begin{array}{cccccccccccccc}
x_{1} & 0 & x_{1} & 0 & 0 & 0 & 0 & 1 & -1 & -1 & 0 & -x_{1} & 0 & 0 \\
x_{2} & 0 & 0 & 0 & 0 & 0 & 0 & 1 & 0 & -1 & 0 & -x_{1} & 0 & 0 \\
0 & x_{1} & 0 & 0 & 0 & 0 & 0 & 1 & 0 & -1 & 0 & -x_{1} & 0 & 0 \\
0 & x_{2} & 0 & 0 & 0 & 0 & 0 & 1 & 0 & 0 & 0 & 0 & 0 & 0 \\
0 & 0 & 1 & 0 & 0 & 0 & 0 & 0 & 0 & 0 & -x_{1} & 1 & 0 & -1 \\
0 & 0 & x_{2} & 0 & 0 & 0 & 0 & 0 & 0 & 0 & 0 & x_{1} & 0 & 0 \\
0 & 0 & 0 & 1 & 0 & 0 & 0 & 1 & 0 & -1 & 0 & -x_{1} & 0 & 0 \\
0 & 0 & 0 & x_{1} & 0 & 0 & 0 & 0 & 0 & 0 & -x_{1} & 1 & 0 & -1 \\
0 & 0 & 0 & x_{2} & 0 & 0 & 0 & 0 & 0 & 0 & 0 & 1 & 0 & 0 \\
0 & 0 & 0 & 0 & 1 & 0 & 0 & 0 & -x_{1} & 0 & 0 & -2 & 0 & 0 \\
0 & 0 & 0 & 0 & x_{2} & 0 & 0 & 0 & 0 & 0 & 0 & -2 x_{2} & 0 & -x_{1} \\
0 & 0 & 0 & 0 & 0 & 1 & 0 & -1 & -1 & 0 & 0 & 0 & 0 & 0 \\
0 & 0 & 0 & 0 & 0 & x_{1} & 0 & 0 & -x_{1} & 0 & 0 & -1 & 0 & 0 \\
0 & 0 & 0 & 0 & 0 & x_{2} & 0 & 0 & 0 & -x_{2} & -x_{2} & -1 & 0 & -1 \\
0 & 0 & 0 & 0 & 0 & 0 & 1 & -1 & 0 & 1 & 0 & x_{1} & 0 & 0 \\
0 & 0 & 0 & 0 & 0 & 0 & x_{1} & 0 & 0 & 0 & x_{1} & -1 & 0 & 1 \\
0 & 0 & 0 & 0 & 0 & 0 & x_{2} & 0 & 0 & 0 & 0 & -1 & 0 & 0 \\
0 & 0 & 0 & 0 & 0 & 0 & 0 & x_{1} & 0 & 0 & 0 & -1 & 0 & 0 \\
0 & 0 & 0 & 0 & 0 & 0 & 0 & x_{2} & 0 & -x_{2} & -x_{2} & -1 & 0 & 0 \\
0 & 0 & 0 & 0 & 0 & 0 & 0 & 0 & x_{2} & 0 & 0 & 0 & 0 & -1 \\
0 & 0 & 0 & 0 & 0 & 0 & 0 & 0 & 0 & x_{1} & 0 & 0 & 0 & -1 \\
0 & 0 & 0 & 0 & 0 & 0 & 0 & 0 & 0 & 0 & 1 & -x_{1} & 0 & 0 \\
0 & 0 & 0 & 0 & 0 & 0 & 0 & 0 & 0 & 0 & 0 & 0 & 1 & -x_{1}
\end{array}\right) \in \mathbb{R}^{23 \times 14}
$$

For brevity, let us use the following sparse matrix notation: $A=\{\ldots,(i, j$ : $\left.\left.a_{i j}\right), \ldots\right\}$ denotes that the entry of matrix $A$ in the $i$ th row and $j$ th column is $a_{i j}$. Using this notation, the two annihilators $C_{a}^{(7)}$ and $\aleph_{\pi_{a}}^{(7)}$ of function $\pi_{a}^{(7)}$ can be given as follows:

$C_{a}^{(7)}=\left\{\left(1,1: x_{1}\right),(1,3:-1),\left(2,1: x_{2}\right),(2,4:-1),\left(3,2: x_{2}\right),(3,5:-1)\right.$, $\left(4,3: x_{1}\right),(4,6:-1),\left(5,3: x_{2}\right),(5,7:-1),\left(6,4: x_{2}\right),(6,8:-1),\left(7,5: x_{2}\right)$, $(7,9:-1),\left(8,2:-2 x_{1}\right),(8,10:-1),\left(9,1: x_{1}\right),\left(9,2:-x_{1}-x_{2}\right),(9,7:$ $\left.x_{1}\right),(9,11:-1),\left(10,1: 2 x_{2}\right),\left(10,2:-2 x_{2}\right),\left(10,7: 2 x_{2}\right),(10,12:-1)$, $\left(11,10: x_{1}\right),(11,13:-1),(11,21: 1),\left(12,10: x_{2}\right),(12,14:-1),(12,28: 1)$, $\left(13,11: x_{2}\right),(13,15:-1),(13,29: 1),\left(14,12: x_{2}\right),(14,16:-1),(14,30: 1)$, $\left(15,2:-x_{1}\right),(15,17: 1),\left(16,2:-x_{2}\right),(16,18: 1),\left(17,1: x_{1}\right),\left(17,2:-x_{1}\right)$, $\left(17,7: x_{1}\right),(17,19: 1),\left(18,1: x_{2}\right),\left(18,2:-x_{2}\right),\left(18,7: x_{2}\right),(18,20: 1)$, $\left(19,17:-x_{1}\right),(19,21:-1),\left(20,17:-x_{2}\right),(20,22:-1),\left(21,18:-x_{2}\right)$, $(21,23:-1),\left(22,21: x_{1}\right),(22,24:-1),\left(23,21: x_{2}\right),(23,25:-1),(24,22:$ $\left.x_{2}\right),(24,26:-1),\left(25,23: x_{2}\right),(25,27:-1),\left(26,19:-x_{1}\right),(26,28:-1)$, $\left(27,19:-x_{2}\right),(27,29:-1),\left(28,20:-x_{2}\right),(28,30:-1),\left(29,28: x_{1}\right)$, $(29,31:-1),\left(30,28: x_{2}\right),(30,32:-1),\left(31,29: x_{2}\right),(31,33:-1),(32,30:$ $\left.\left.x_{2}\right),(32,34:-1)\right\} \in \mathbb{R}^{32 \times 34}$

and

$\aleph_{\pi_{a}}^{(7)}=\left\{\left(1,1: x_{2}\right),\left(1,2:-x_{1}\right),\left(2,3: x_{2}\right),\left(2,4:-x_{1}\right),\left(3,4: x_{2}\right),\left(3,5:-x_{1}\right)\right.$, $\left(4,6: x_{2}\right),\left(4,7:-x_{1}\right),\left(5,7: x_{2}\right),\left(5,8:-x_{1}\right),\left(6,8: x_{2}\right),\left(6,9:-x_{1}\right),(7,2:$ 
$\left.x_{1}\right),(7,4:-1),\left(8,4: x_{1}\right),(8,7:-1),\left(9,5: x_{1}\right),(9,8:-1),\left(10,1:-x_{1}\right)$, $\left(10,2: x_{1}-x_{2}\right),\left(10,7:-x_{1}\right),(10,18: 1),(10,19:-1),\left(11,10: x_{2}\right),(11,11:$ $\left.-x_{1}\right),(11,22:-1),(11,28: 1),\left(12,11: x_{2}\right),\left(12,12:-x_{1}\right),(12,23:-1)$, $(12,29: 1),\left(13,13: x_{2}\right),\left(13,14:-x_{1}\right),(13,25:-1),(13,31: 1),(14,14:$ $\left.x_{2}\right),\left(14,15:-x_{1}\right),(14,26:-1),(14,32: 1),\left(15,15: x_{2}\right),\left(15,16:-x_{1}\right)$, $(15,27:-1),(15,33: 1),\left(16,1: x_{1}\right),\left(16,2:-x_{1}\right),\left(16,7: x_{1}\right),(16,11:-1)$, $(16,18:-1),\left(17,11: x_{1}\right),(17,14:-1),(17,22: 1),\left(18,12: x_{1}\right),(18,15:$ $-1),(18,23: 1),\left(19,17: x_{2}\right),\left(19,18:-x_{1}\right),\left(20,19: x_{2}\right),\left(20,20:-x_{1}\right)$, $\left(21,21: x_{2}\right),\left(21,22:-x_{1}\right),\left(22,22: x_{2}\right),\left(22,23:-x_{1}\right),\left(23,24: x_{2}\right)$, $\left(23,25:-x_{1}\right),\left(24,25: x_{2}\right),\left(24,26:-x_{1}\right),\left(25,26: x_{2}\right),\left(25,27:-x_{1}\right)$, $\left(26,22: x_{1}\right),(26,25:-1),\left(27,23: x_{1}\right),(27,26:-1),\left(28,28: x_{2}\right),(28,29:$ $\left.-x_{1}\right),\left(29,29: x_{2}\right),\left(29,30:-x_{1}\right),\left(30,31: x_{2}\right),\left(30,32:-x_{1}\right),\left(31,32: x_{2}\right)$, $\left(31,33:-x_{1}\right),\left(32,33: x_{2}\right),\left(32,34:-x_{1}\right),\left(33,29: x_{1}\right),(33,32:-1)$, $\left.\left(34,30: x_{1}\right),(34,33:-1)\right\} \in \mathbb{R}^{34 \times 34}$,

(A.9b)

Using Procedure 1), we obtained the following annihilator for $\pi_{a}^{(7)}$ :

$N_{a}^{(7)}=\left\{\left(1,1: x_{1}\right),(1,18: 1),(1,19:-1),(1,20:-1),(1,24:-1)\right.$, $(1,25:-1),\left(2,1: x_{2}\right),(2,18: 1),(2,20:-1),(2,25:-1),\left(3,2: x_{1}\right)$, $(3,18: 1),(3,20:-1),(3,25:-1),\left(4,2: x_{2}\right),(4,18: 1),(5,3: 1)$, $(5,18: 1),(5,19:-1),(5,20:-1),(5,24:-1),(5,25:-1),\left(6,3: x_{1}\right)$, $(6,23: 1),\left(6,24:-x_{1}\right),\left(6,25:-x_{1}\right),\left(6,26:-x_{1}\right),(6,28:-1),(6,29:-1)$, $(6,30:-1),\left(7,3: x_{2}\right),(7,23: 1),\left(7,25:-x_{1}\right),\left(7,26:-x_{1}\right),(7,29:-1)$, $(7,30:-1),(8,4: 1),(8,18: 1),(8,20:-1),(8,25:-1),\left(9,4: x_{1}\right)$, $(9,23: 1),\left(9,25:-x_{1}\right),\left(9,26:-x_{1}\right),(9,29:-1),(9,30:-1),\left(10,4: x_{2}\right)$, $(10,23: 1),\left(10,26:-x_{1}\right),(10,30:-1),(11,5: 1),(11,18: 1),\left(12,5: x_{1}\right)$, $(12,23: 1),\left(12,26:-x_{1}\right),(12,30:-1),\left(13,5: x_{2}\right),(13,23: 1),(14,6: 1)$, $(14,23: 1),\left(14,24:-x_{1}\right),\left(14,25:-x_{1}\right),\left(14,26:-x_{1}\right),(14,28:-1)$, $(14,29:-1),(14,30:-1),\left(15,6: x_{2}\right),(15,24: 1),(16,7: 1),(16,23: 1)$, $\left(16,25:-x_{1}\right),\left(16,26:-x_{1}\right),(16,29:-1),(16,30:-1),\left(17,7: x_{1}\right)$, $(17,24: 1),\left(18,7: x_{2}\right),(18,25: 1),(19,8: 1),(19,23: 1),\left(19,26:-x_{1}\right)$, $(19,30:-1),\left(20,8: x_{1}\right),(20,25: 1),\left(21,8: x_{2}\right),(21,26: 1),(22,9: 1)$, $(22,23: 1),\left(23,9: x_{1}\right),(23,26: 1),\left(24,9: x_{2}\right),(24,27: 1),(25,10: 1)$, $(25,18:-2),(25,20: 2),(25,25: 2),\left(26,10: x_{1}\right),(26,23:-2),(26,25:$ $\left.2 x_{1}\right),\left(26,26: 2 x_{1}\right),(26,29: 2),(26,30: 2),\left(27,10: x_{2}\right),(27,23:-2)$, $\left(27,26: 2 x_{1}\right),(27,30: 2),(28,11: 1),(28,18:-1),(28,19:-1),(29,11:$ $\left.x_{1}\right),(29,23:-1),\left(29,26: x_{1}\right),(29,28:-1),(29,30: 1),\left(30,11: x_{2}\right)$, $(30,23:-1),(30,29:-1),(31,12: 1),(31,20:-2),\left(32,12: x_{1}\right),(32,29:$ $-2),\left(33,12: x_{2}\right),(33,30:-2),(34,13: 1),(34,23:-3),\left(34,25: 3 x_{1}\right)$, 
$\left(34,26: 3 x_{1}\right),(34,29: 3),(34,30: 3),\left(35,13: x_{1}\right),(35,24:-3),(36,13:$ $\left.x_{2}\right),(36,25:-3),(37,14: 1),(37,23:-2),\left(37,26: 2 x_{1}\right),(37,28:-1)$, $(37,30: 2),\left(38,14: x_{1}\right),(38,25:-2),(38,31:-1),\left(39,14: x_{2}\right),(39,26:$ $-2),(39,32:-1),(40,15: 1),(40,23:-1),(40,29:-2),\left(41,15: x_{1}\right)$, $(41,26:-1),(41,32:-2),\left(42,15: x_{2}\right),(42,27:-1),(42,33:-2),(43,16$ : 1), (43,30:-3), (44,16: $\left.x_{1}\right),(44,33:-3),\left(45,16: x_{2}\right),(45,34:-3)$, $(46,17: 1),(46,18:-1),(46,20: 1),(46,25: 1),\left(47,17: x_{1}\right),(47,23:-1)$, $\left(47,25: x_{1}\right),\left(47,26: x_{1}\right),(47,29: 1),(47,30: 1),\left(48,17: x_{2}\right),(48,23:-1)$, $\left(48,26: x_{1}\right),(48,30: 1),\left(49,18: x_{1}\right),(49,23:-1),\left(49,26: x_{1}\right),(49,30: 1)$, $\left(50,18: x_{2}\right),(50,23:-1),\left(51,19: x_{1}\right),(51,28:-1),\left(52,19: x_{2}\right),(52,29:$ $-1),\left(53,20: x_{1}\right),(53,29:-1),\left(54,20: x_{2}\right),(54,30:-1),(55,21: 1)$, $(55,23:-1),\left(55,25: x_{1}\right),\left(55,26: x_{1}\right),(55,29: 1),(55,30: 1),(56,21:$ $\left.x_{1}\right),(56,24:-1),\left(57,21: x_{2}\right),(57,25:-1),(58,22: 1),(58,23:-1)$, $\left(58,26: x_{1}\right),(58,30: 1),\left(59,22: x_{1}\right),(59,25:-1),\left(60,22: x_{2}\right),(60,26:$ $-1),\left(61,23: x_{1}\right),(61,26:-1),\left(62,23: x_{2}\right),(62,27:-1),\left(63,24: x_{2}\right)$, $\left(63,25:-x_{1}\right),\left(64,25: x_{2}\right),\left(64,26:-x_{1}\right),\left(65,26: x_{2}\right),\left(65,27:-x_{1}\right)$, $\left(66,28: x_{1}\right),(66,31:-1),\left(67,28: x_{2}\right),(67,32:-1),\left(68,29: x_{1}\right),(68,32:$ $-1),\left(69,29: x_{2}\right),(69,33:-1),\left(70,30: x_{1}\right),(70,33:-1),\left(71,30: x_{2}\right)$, $(71,34:-1),\left(72,31: x_{2}\right),\left(72,32:-x_{1}\right),\left(73,32: x_{2}\right),\left(73,33:-x_{1}\right)$, $\left.\left(74,33: x_{2}\right),\left(74,34:-x_{1}\right)\right\} \in \mathbb{R}^{74 \times 34}$

(A.10) 